\title{
Universiteit
}

Leiden

The Netherlands

\section{Targeting Polymeric Nanobiomaterials as a Platform for Cartilage Tissue Engineering}

Garcia Couce, J.; Almirall, A.; Fuentes, G.; Kaijzel, E.; Chan, A.; Cruz, L.J.

\section{Citation}

Garcia Couce, J., Almirall, A., Fuentes, G., Kaijzel, E., Chan, A., \& Cruz, L. J. (2019). Targeting Polymeric Nanobiomaterials as a Platform for Cartilage Tissue Engineering. Current

Pharmaceutical Design, 25(17), 1915-1932. doi:10.2174/1381612825666190708184745

Version: $\quad$ Not Applicable (or Unknown)

License: $\quad$ Leiden University Non-exclusive license

Downloaded from: https://hdl.handle.net/1887/121577

Note: To cite this publication please use the final published version (if applicable). 


\title{
Targeting Polymeric Nanobiomaterials as a Platform for Cartilage Tissue Engineering
}

\author{
Jomarien García-Couce ${ }^{\mathrm{a}, \mathrm{b}}$, Amisel Almirallb,c ${ }^{\mathrm{b}}$, Gastón Fuentes ${ }^{\mathrm{b}, \mathrm{c}}$, Eric Kaijzel ${ }^{\mathrm{b}}$, Alan Chan ${ }^{\mathrm{d}}$ and Luis J. \\ $\mathrm{Cruz}^{* \mathrm{~b}}$, \\ ${ }^{a}$ Department of Polymeric Biomaterials, BIOMAT, University of Havana, Cuba \\ ${ }^{\mathrm{b}}$ Translational Nanobiomaterials and Imaging (TNI) group, Radiology department, Leiden University Medical Centrum, \\ Leiden, The Netherlands \\ 'Department of Ceramics and Metallic Biomaterials, BIOMAT, University of Havana, Cuba; ${ }^{\text {dPercuros B.V., Zernikedreef }}$ \\ 8, 2333 CL Leiden, The Netherlands
}

\begin{abstract}
Articular cartilage is a connective tissue structure that is found in anatomical areas that are important for the movement of the human body. Osteoarthritis is the ailment that most often affects the articular cartilage. Due to its poor intrinsic healing capacity, damage to the articular cartilage is highly detrimental and at present the reconstructive options for its repair are limited. Tissue engineering and the science of nanobiomaterials are two lines of research that together can contribute to the restoration of damaged tissue. The science of nanobiomaterials focuses on the development of different nanoscale structures that can be used as carriers of drugs / cells to treat and repair damaged tissues such as articular cartilage. This review article is an overview of the composition of articular cartilage, the causes and treatments of osteoarthritis, with a special emphasis on nanomaterials as carriers of drugs and cells, which reduce inflammation, promote the activation of biochemical factors and ultimately contribute to the total restoration of articular cartilage.
\end{abstract}

Keywords: Targeting platform, polymeric nanobiomaterials, cartilage tissue engineering.

\section{INTRODUCTION}

Articular cartilage is a highly specialized tissue that protects the ends of the bones that make up the joint. Its function is to reduce friction and offer a lubricated surface to prevent joint wear. Like other connective tissues, it is made up of cells and an extracellular matrix, but it differs from the rest of the connective tissues because it lacks blood vessels and nerves and receives its nutrition only by diffusion of molecules of synovial fluid that crosses its surface [1]. Compared to other connective tissues, cartilage has a very slow turnover in its extracellular matrix (ECM) [2,3]. Structurally, cartilage has only one type of cell, the chondrocytes; and extracellular matrix, which is composed mainly of collagen and proteoglycans. However, its structure is divided into three or four layers according to previous reports from different authors. $[4,5]$.

Damage to the articular cartilage begins as a point defect, progressing gradually to a total defect that encompasses all the layers of the damaged part, with the lesion coming into contact with the surrounding bone. Due to the very slow renewal of the matrix, the minimal proliferation capacity of the chondrocytes and poor blood supply, the articular cartilage cannot repair itself $[6,7]$.

Despite all the knowledge that has been acquired over the years from studies on osteoarthritis (OA), drugs capable of modifying the progression of the disease are not yet available in the market. Although some drugs have been developed that act on specific elements of the progression of the disease of OA, its clinical use is not viable due to the large number of serious side effects. Therefore, clinical practice is based on a symptomatic treatment to relieve pain and to surgically replace joints with OA at the terminal stage [8]. Although significant advances have been made in orthopedic surgery, the treatment of cartilage damage remains a challenge. Due to the existence of numerous limitations such as graft instability, calcification and applications restricted to focal lesions, new therapeutic strategies based on cell therapy and tissue engineering have been developed [9]. 
With tissue engineering, it is possible to design an artificial cartilage from the sowing of chondrogenic cells in biodegradable scaffolds. This methodology provides an alternative strategy that can repair defects in the area of articular cartilage or meniscus restoring the function of loading the joint and relieving pain [10]. The annual growth rate that cartilage therapy based on tissue engineering undergoes is $12.5 \%$ and is expected to be maintained until 2020. This will result in an expected growth from $\$ 255$ million in 2015 to $\$ 460$ million by 2020 . It is evident that the market potential in the regeneration of cartilage tissue is large and is expected to increase over the years [11].

Nanomaterials are characterized by their nanoscale dimension, which allows them to develop critical physical and chemical characteristics that improve their performance and make them suitable for a wide range of applications. In the biomedical field, nanobiomaterials have been used for the controlled administration of drugs, images of specific sites, exploration of DNA structures, biomolecular detection, administration of genes and, more recently in tissue engineering (TE). Nanomaterial science has introduced new methods to improve the quality of scaffolds used TE and regenerative medicine. The scaffolds can now be designed at the nanoscale, using different techniques and also incorporating nanoparticles [12-15].

In this review, we focus on the different applied nanosystems for cartilage tissue engineering. We discuss the advances of nanoformulations in the application of bioactive molecules in the target zone, specifically cartilage.

\section{COMPOSITION AND STRUCTURE OF ARTICULAR CARTILAGE}

Of the major components of articular cartilage in the hydrated state, water forms the greater part (68$85 \%)$ followed by collagen (10-20\%) and proteoglycans (5-10\%) [16].

According to the percentages described, the water distributed in the interstices of the joint tissue is the most abundant component [17], and the amount present is associated with the concentration of proteoglycans [18] and the amount of dissolved ions in solution that forms the organization of the collagenous matrix [19]. The relationship and types of collagens that make up the ECM in normal and damaged articular cartilage have been studied in depth [19-27]. Of the different types of collagens identified at least five are present in the articular cartilage $[23,28]$. The extracellular matrix is made up of type II, IX and XI collagens; type II provides the basic architecture of the tissue [16, 29] and XI is probably copolymerized with type II collagen in the matrix [23, 25]. Type VI collagen increases in the pericellular region of the middle and deep zones of the articular cartilage, but is reduced in the superficial layer in damaged cartilage [30]. Covalently attached to the surface of type II collagen fibrils is type IX collagen, which represents only $1 \%$ of total cartilage collagen [22, 24, 27]. Finally, it is worth mentioning the type $\mathrm{X}$ collagen that is only found in the calcified area of the articular cartilage.

\section{Types of cartilage}

Vertebrate cartilage(s) is subdivided into types primarily on the basis of histology. On histological grounds, cartilage is $[2,3,31,32]$ :

$\square$ hyaline, if the matrix is composed predominantly of glycosaminoglycans;

$\square$ elastic, if elastic fibers are present in the extracellular matrix;

$\square$ or fibrous (fibrocartilage), if the matrix has an enriched collagenous fiber content, including type I collagen.

\section{Hyaline cartilage}

Of the cartilage subtypes described, the hyaline cartilage of the arthritic articular surfaces is the most frequent and best characterized [1,33]. In the mammalian kingdom, the hyaline cartilage constitutes the embryonic models of the endochondral bones and portions of the laryngeal cartilage; the elastic cartilage is composed of the pinna, larynx, epiglottis and intervertebral discs [3, 31, 34]. 
Hyaline cartilage is the cartilaginous tissue most abundant in the human body and is characterized by having a bluish hue and is opalescent. The ventral aspects of the ribs, the trachea and the bronchial tree also exist on the articular surface of the bones [2].

In bones, osteons are the basic structural unit of compact bone; similarly in the cartilage there are basic structures known as chondrons and have been recognized for a century only. A chondrona consists of a chondrocyte nest and its collective pericellular matrix [3, 35].

In the research carried out by $\mathrm{C}$. Anthony Poole and his team in Auckland, New Zealand, it has been possible to know in detail the characteristics and properties of the chondrons in the last two decades. The results of extensive analysis on these basic structures allow us to say today that $[35,36]$ :

$\square$ pericellular capsules of fine fibrils are characterized by deep but not superficial canine tibial articular cartilage;

$\square$ adult human articular cartilage contains similar chondrones;

$\square$ chondrones can be physically isolated as intact structural units;

$\square$ chondrones are rich in proteoglycans;

$\square$ type VI collagen is present in the pericellular capsule of isolated canine tibial chondrones, which suggests that chondrones resist loading in dynamic compression; and

$\square$ chondrones isolated from human or canine osteoarthritic cartilage are defective in collagen and proteoglycan.

It is believed that the diversity of growth mechanisms explains the success of cartilage as an embryonic skeletal tissue. In contrast to bone that grows only appositive, the growth of cartilage is both internal by the proliferation of chondroblasts/chondrocytes and deposition of the extracellular matrix, as appositive by joining to the surfaces of the cartilage. The cartilage is an ideal tissue that can resist the compression given by the large volumes of fluid joined through cartilaginous matrices. This property increases its usefulness in growing organisms and makes it an ideal tissue to join and lubricate human surfaces. [3].

\section{Elastic cartilage}

Another type of cartilage mentioned is elastic cartilage, found in areas of the outer ear, the Eustachian tube, the epiglottis and parts of the larynx. It fulfills the function of guaranteeing the permeability of the luminaire of the tubes that are surrounded by this variety of cartilage. In the elastic cartilage, the cells that deposit extracellular matrices rich in elastin form a recognizable subset, allowing to identify and define said cartilage [2].

The presence of elastic fibers and elastin has been observed in cartilage, bones and joints through the use of specific histochemical and immunohistochemical procedures. The presence of a certain amount of elastic fibers in some cartilages leads to its being called histologically as elastic cartilage, and the cartilaginous elements as elastic cartilages. Compared with hyaline cartilage, elastic cartilage is typically more cellular and the chondrocytes that make it up, although they have a similar cytomorphology, are completely randomized $[1-3,32]$.

The distribution of the elastic fibers is very variable depending on the type of cartilage in which they are found. In the joints they can be found in the fibrous articular tissue; in the hyaline and fibrous cartilages, they are observed in the fibrous perichondria. In bones, the outer fibrous layer of the periosteum has elastic fibers in its structure; however, they are not found in the inner cellular (cambial) layer [2, 3, 32, 37, 38].

With a routine $H \& E$ histological technique, elastin fibers can be identified by the amorphous blue tone that is distinct from the vitreous appearance of the hyaline cartilage. However, these fibers can best be identified with a special histochemical technique using sliver-based stains [1].

\section{Fibrous cartilage}

The fibrous cartilage also referred to as fibrous-elastic cartilage is organized in such a way that the concentric layers of fibrous tissue overlap the surface when viewed from above. The matrix that composes it is more fibrillar and eosinophilic than that of the hyaline cartilage and the chondrocytes present are smaller 
and distributed in a more casual way. A trichrome stain, which dyes the collagen in a deep blue while staining the cytoplasm and other red-colored proteins, is useful to demonstrate the extensive unidirectional arrangement of collagen that imparts resistance to tensile forces. It is found in different places such as the meniscus of the knee and labrum of the shoulder and hip joints, but the fibrous annulus of the intervertebral disc represents the most prevalent fibrocartilage in the human body $[1,33]$

\section{Cartilage cells: Chondrocytes}

Chondrocytes are specialized and isolated cells, which produce and maintain the ECM of cartilage; they occupy only 5\% of the matrix [39]. Depending on the area of the cartilage in which they are found, they have different shapes and sizes, as well as a diverse metabolic activity, which is conditioned by the unique physiological and mechanical environment in which they develop [16, 40]

Biological processes that are important for homeostasis and cartilage repair to occur, including cell attachment, growth, differentiation and survival are regulated by the interactions between chondrocytes and the ECM. The cell-matrix interactions linked to the regulation of these processes are mediated by different molecules, among them the integrin family of cell surface receptors seems to play an important role [41].

\section{Extracellular matrix}

Although it has been reiterated that cartilage is a complex tissue, its natural division is simple: cells (chondrocytes) + ECM. The cartilage, the collagen that forms it and the rest of the components are suspended or dissolved in the fluid that constitutes the extracellular matrix. In connective tissues, the ECM is formed by a three-dimensional crosslinked network of insoluble collagen fibers in which are other components such as proteoglycans and glycoproteins that are more soluble and can be entangled, integrated or even chemically bound in it $[29,42]$.

\section{Collagens}

The proteins that form a triple helix characteristic of three polypeptide chains are called "collagen". The family of collagens that make up the extracellular matrix form these supramolecular structures, although their size, function, and tissue distribution vary considerably [42]. Collagen type II is predominant. Its polymers are the fibrils that form the basic framework of the tissue. Of the less abundant collagen species in cartilage, types IX and XI are, like type II collagen, specific to cartilage. Knowledge of the molecular mechanisms by which collagen architecture is established by chondrocytes, remodeled during life and irreversibly damaged in joint disease, is essential to understand the pathogenesis of osteoarthritis [23]. The main characteristics of these components are [2,43]:

Collagen Type II $\left(\mathrm{Mw}_{\mathrm{w}}=425 \mathrm{kDa}\right)$ : Triple-helical glycoprotein has a fibrillar molecule with a periodicity of $67 \mathrm{~nm}$. It is predominantly present collagen of cartilage and intervertebral disc, has tensile strength, supports chondrocyte adhesion and induces phenotypic differentiation of cells.

$\square$ Collagen Type IX $(\mathrm{Mw}=222 \mathrm{kDa})$ : Disulphide-bonded heterodimer. It is present in cartilage and intervertebral disc. It bridges collagen fibrils with other macromolecules, associates with the surface of type II collagen and participates in the formation of type II collagen

$\square$ Collagen Type XI (Mw = $545 \mathrm{kDa})$ : Minor fibrillar collagen. It forms heterotypic fibrils with type II and IX collagens. It is predominantly present in cartilaginous tissue and also expressed by nonchondrogenic tissues.

The other collagens (Type VI, X, XII and XIV), minorities in the hyaline cartilage, are part of the macroand microfibrils cross-linked on the surface to provide structural support to the cartilage [2, 44].

Collagen type III is consistently detected by immunofluorescence in samples of normal and osteoarthritic human articular cartilage and it is used as a reference to evaluate possible osteoarthritis because of its ability 
to cross-link and increase the concentration on the surface and upper middle zone and to be synthesized by the chondrocytes in the absence of collagen I expression $[26,45,46]$.

\section{Proteoglycans}

In the composition of the articular cartilage, there is a great variety of non-collagens proteins and polysaccharides that vary in their abundance and structure according to the anatomical site or the age of the person. Many of the molecules are proteoglycans, which carry chains of glycosaminoglycans, while others are glycoproteins or even non-glycosylated proteins. Among the non-collagen proteins, aggrecan is of great importance. The high content of aggrecan in the extracellular matrix of cartilage is responsible for the unique ability of the tissue to resist compression in a specialized manner [2, 44, 47].

Additionally, human cartilage contains three dermatan sulfate proteoglycans and two potential keratan sulfate proteoglycans. The presence of the above mentioned ECM's proteins in articular cartilage is important for normal articular cartilage function. The extracellular matrix of other cartilage types contains numerous other proteins (e.g. cartilage matrix protein) that are neither collagens nor proteoglycans, and several of these are thought to play a structural role in the matrix. Of special relevance for cartilage tissue is hyaluronan and S-100 [2, 3, 16, 39, 48]. The most important proteoglycans are [2, 44]:

Aggrecan $(\mathrm{Mw}=205 \mathrm{kDa})$ : Large chondroitin sulphate proteoglycan. It comprises chondroitin sulphate $(87 \%)$, keratan sulphate $(6 \%)$ and cartilage dry weight $(10 \%)$. It is not found in bones and interacts with hyaluronan. It has a large number of fixed negatively charged side groups and is responsible for high osmotic pressure. Its primary role is to swell and hydrate collagen framework.

$\square$ Link Protein $(\mathrm{Mw}=45 \mathrm{kDa})$ : Glycoprotein. It binds to aggrecan and hyaluronan.

Matrix Gla Protein $(\mathrm{Mw}=12 \mathrm{kDa})$ : Glycoprotein. It is present in bone and cartilage and acts as an inhibitor of calcification.

$\square$ Biglycan: It is predominantly in small proteoglycan of cartilage and leucine-rich proteoglycan. It is relatively abundant in the bone mineral compartment.

$\square$ Fibromodulin $(\mathrm{Mw}=42 \mathrm{kDa})$ : Small chondroitin sulphate/dermatan sulphate proteoglycan. It is found in a variety of tissues and abundant in cartilage. It compromises $0.1-0.3 \%$ of cartilage wet weight. It binds to types I and II collagen in vitro and inhibits collagen fibrillogenesis in vitro.

$\square$ Cartilage Matrix Protein $(\mathrm{MW}=54 \mathrm{kDa})$ : Disulphide-bonded multimer. It is a major component of non-articular cartilage and is present mostly in tracheal (5\% wet weight), nasal, and epiphyseal cartilage.

$\square$ Hyaluronan $(0.1<\mathrm{Mw}<1 \mathrm{MDa})$ : High molecular mass polysaccharide. It is found in copolymer of glucuronic acid and $\mathrm{N}$-acetylglucosamine. It is present in a number of tissues. It is synthesized in the plasma membrane of cells and catabolized preferentially at local sites. It plays an important role during tissue development and differentiation and is used for water and plasma protein homeostasis [49].

$\square \mathrm{S} 100$ protein $(\mathrm{Mw}=21 \mathrm{kDa})$ : Acidic calcium-binding protein. It is present in various conformations. It modulates a wide range of cellular processes and is used as a chondrocyte marker protein. It distinguishes cartilage from bone tissue.

\section{Structure of the cartilage}

Cartilage is one of the most fascinating areas of the human body when it comes to analyzing its threedimensional structure. Still undecided between pointing three or four layers when making a cross section, most of the researchers in this field prepare scaffolds with three layers in which the deepest is always doped with a bioactive inorganic filler that gives that separation in two layers: the deep and the subchondral [5052].

The boundary between the first three zones of the cartilage is established arbitrarily according to the changes observed in the morphology, position and distribution of the components of the matrix. However, the deep and calcified zones are separated by a distinct mineralization front known as the tidemark. The tidemark is a unique histological feature of articular cartilage and is not present in other hyaline cartilage 
[1]. At the microscopic levels it varies significantly with depth and can be separated into four imprecisely defined zones [7, 16, 44]:

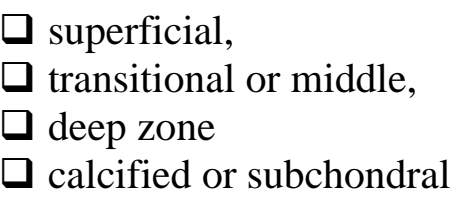

The superficial zone is formed by two layers, an upper one composed of fine fibrils with little polysaccharide and the other one that almost completely lacks chondrocytes that cover the surface of the joint. It is a highly important area in the resistance to tensile forces that are generated during the deformation of the surface under load [53]. The damage in its structure can contribute to the development of osteoarthritis by changing the mechanical behavior and possibly releasing molecules that stimulate an immune or inflammatory response $[16,54]$.

The transition zone is larger than the superficial zone and differs in terms of cellularity and organization. The chondrocytes of this zone are more spheroidal, they have a lower density and are embedded in an extensive extracellular matrix. The concentration of proteoglycans is higher and the collagen fibrils are larger in diameter and are arranged in a more random way [54].

In deep zone, the cells are distributed perpendicular to the surface and their shape is more rounded. This zone contains the largest diameter collagen fibrils, located equally perpendicular to the surface, and the highest concentration of proteoglycans $[16,44,55]$.

As explained above there is some hesitation in dividing the cartilage into three [50-52] or four [2, 16, 44] zones. But the truth is that all authors agree that in the deepest layer there is an area which is closest to the bone and it has been called subchondral zone. Some authors consider this as a particular region for some of its characteristics mostly related with cell density and type, protein concentration and mechanical properties $[1,29,56]$.

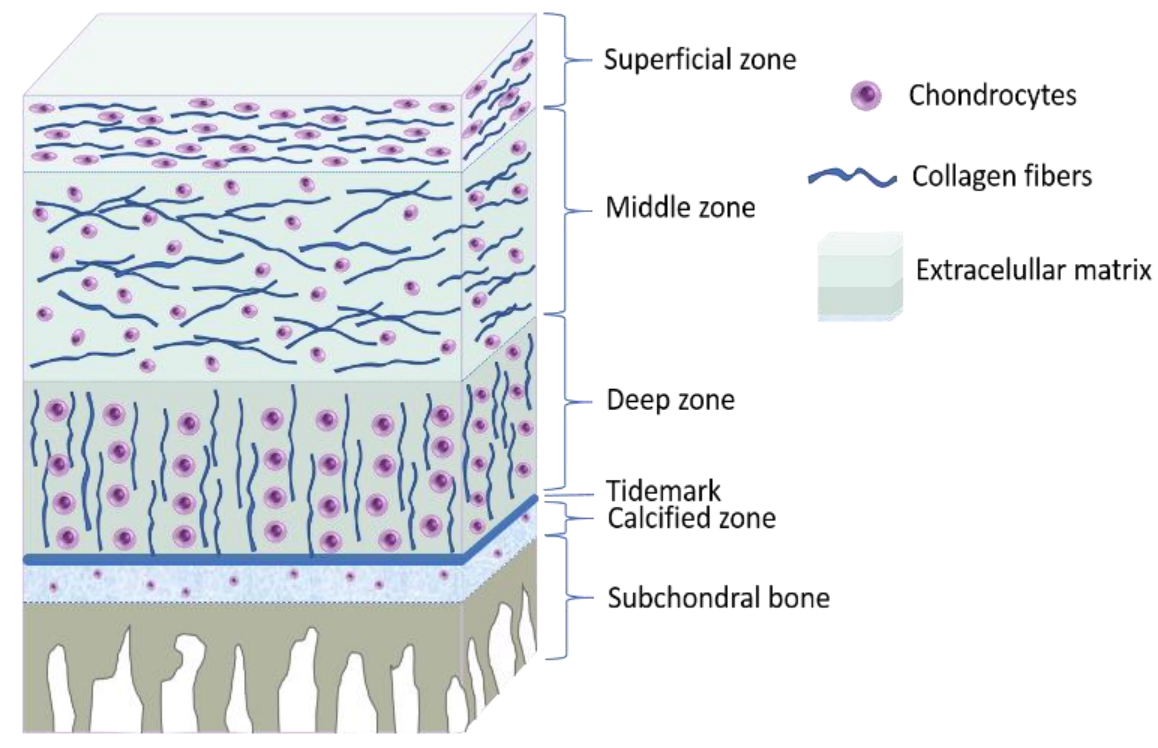

Fig. (1). Structure and different zones of the normal cartilage tissue.

\section{CARTILAGE DAMAGE}

When you think about joint damage, whether it is on shoulders, elbows, hips or knees, the first word that comes to mind is osteoarthritis, probably the best known of all the pathologies related to this type of tissue. 
OA remains a public health problem of global importance, as outlined in several reports found at international academic and health database [57-62]. To mention some numbers, OA affects approximately $18 \%$ of women and $10 \%$ of men over 60 years of age. This represents a total of 240 million people, who carry a considerable morbidity, including disability and a low quality life. It also highlights the lack of effective treatment strategies in this common chronic condition [61, 62]. In fact, there are currently no approved medications that can prevent, stop or even restrict the progression of OA. In addition, available medications that promise to alleviate OA pain have a number of risk/benefit considerations [62].

\section{Osteoarthritis}

Normally, every specialist defends his/her field as of supreme importance, for improving humans' life quality and increasing their life expectation, but osteoarthritis and diabetes mellitus are probably the most immediate challenges of medical and biomedical research in the $21^{\text {st }}$ century. And it is said immediately precisely because they have more possibilities of finding solution to than for example cancer, acquired immune deficiency syndrome (AIDS), and other important series of pathologies whose cure will take much longer time to find.

Historically, OA has been defined as a non-inflammatory degenerative arthritis with loss of cartilage and narrowing of the joint space. "Wear" arthritis is a commonly used phrase; [58]. However, currently this definition is obsolete since OA is a multifactorial and highly complex disease that involves the entire synovial joint, which includes the cartilage, the synovium and the underlying bone, of which there is still much to be identified in terms of the mechanisms underlying the incidence and the progression. The risk factors known are diverse, however, some, such as obesity and joint injuries, can be mitigated by reducing the impact of the disease [63]. OA can be of primary or secondary type according to how joint damage occurs. If it occurs without an inciting cause, it is of the primary type, whereas, if it occurs due to trauma, infection, metabolic, inflammatory or other disease, it is secondary in nature [58, 64]. Trends in OA from 1990 to 2013 showed an increase of $75 \%$, the third most rapidly associated with disability, just behind diabetes at $135 \%$ and dementia at $84 \%$ [62].

In all the countries where the economic burden represented by arthritis for patients and society has been estimated, the results have shown high values. In the US the total cost attributable to arthritis and other rheumatic conditions in 2003 was approximately $\$ 128$ billion, equivalent to $1.2 \%$ of the country's gross domestic product at that time. In 2009, US reported that costs due to hospital expenditures of total knee and hip joint replacements were $\$ 28.5$ billion and $\$ 13.7$ billion, respectively. Following the sequence in this same country, the total expenses in medical care and earnings losses were attributable to arthritis in 2013 of $\$ 303.5$ billion, representing 1\% of the gross domestic product of the United States in that year. The increase in figures observed over a period of 10 years is significant, which demonstrates the high personal and social impact of arthritis [63, 65-67].

Taking the United States as an example, according to numbers reported, OA affects more than 27 million Americans, approximately $15 \%$ of the population, and contributes some 12 million visits per year to hospitals and health centers, demonstrating that it is the most common joint disease in this population. The values are expected to continue rising, mainly due to population aging and not only in the United States [63, $65,66]$.

OA can be defined pathologically, radiographically or clinically. Due to the ease of standardization and acquisition, radiography is often used as the standard for defining the presence and severity of OA using the Kellgren and Lawrence (KL) grading system [64, 68, 69]. This system scales OA severity on a scale of $0-$ 4 with $>2$ defining radiographic $\mathrm{OA}$.

The following three stages are usually recognized for the purposes of identifying the burden of disease from the previous radiographic evaluation [69-71].

Mild: Kellgren \& Lawrence X-ray grade 0 (none) or 1 (doubtful). 
Moderate: Kellgren \& Lawrence X-ray grade 2 (minimal) or 3 (moderate).

Severe: Kellgren \& Lawrence X-ray grade 4 (severe).

The X-rays, despite being a technique with a large number of years of use, are not available in all parts of the world, so a staging system based on symptoms and physical findings would be preferable. Unfortunately, no such instrument has been validated. Systems such as the Joint Alignment and Motion Scale [72], which uses joint mobility and alignment for the clinical staging of osteoarthritis, could be adapted to, but reproducibility is poor and their impact on patients is uncertain [70].

\section{Incidence and prevalence of $\mathrm{OA}$}

It is good to clarify that the estimation of the incidence of osteoarthritis is very complex. The symptoms of the disease, widely discussed and accepted, depend in part on patient referrals before radiographic evaluations or medical criteria that indicate the possible existence of the disease. The symptoms are nonspecific and the clinical definition of OA has not been established yet with total success. Two of the symptoms that occur in any damaged joint are pain in the first instance and inflation [70]. The clinical variants associated with the complexity of symptoms shown by a person with OA are dissimilar, including generalized primary osteoarthritis, erosive inflammatory arthrosis, diffused idiopathic skeletal hyperostosis and chondromalacia patellae.

$\mathrm{OA}$ is a complex response of joint tissues to aging, as well as to genetic and environmental factors. The OA's pathogenesis includes the contribution of biomechanical and metabolic factors which, altering the tissue homeostasis of articular cartilage and subchondral bone, determine the predominance of destructive over productive processes [73]. Due to several factors such as population growth and aging, the increase in overweight and obesity worldwide, as well as the absence of drugs that can cure or modify the progression of the disease, an increase in the prevalence of the disease is expected [60, 74].

The prevalence of OA can vary between countries, regions, according to their socioeconomic status and the site of the affected joint. For example, hip OA is less frequent in South-east Asian populations compared to Caucasian populations; however, knee OA is more prevalent in black people than in Caucasians. In developed countries, population groups with lower income have a higher incidence of osteoarthritis compared to people who receive higher income, possibly due to factors such as inadequate eating habits that increase obesity and other conditions that constitute risk factors of this disease $[62,70]$.

\section{Classification of $\mathrm{OA}$}

One of the ways to classify the type of OA is related to the factors that lead to damage and loss of articular cartilage. When the disease develops due to aging and is not related to systemic factors or certain clinical erosive and hereditary subsets, it is called primary or idiopathic OA. However, when other factors of higher incidence such as endocrine-metabolic, inflammatory or hereditary disorders are evident, it is defined as secondary OA since, although age may be a contributing factor, it is not the cause of the highest incidence [75].

Currently, with respect to the etiopathogenesis of OA there are two main categories. The first includes damage caused by common abnormalities of weight load or load transmission through the joint, either in terms of distribution of load forces or intensity of such forces. In this category, the rheological properties of the cartilage and the joint capsules are considered normal. Acetabular dysplasias or Legg-Calvé-Perthes disease could be mentioned within this group. The second category includes groups whose damages are associated with factors that involve abnormal rheological properties of the joints as an example; it can show the cartilage in which deposits of metabolites damage the matrix, as it occurs in hemochromatosis and ochronosis. As the additional causes of failure are better understood, more and more cases of osteoarthritis are included in this second category of etiology [75].

\section{Risk factors for $\mathrm{OA}$}


The risk factors in OA are diverse and mostly conditioned by the aging process of human beings. It is one of the causes that qualify OA as the disease of old age. But other factors are recognized like sex, trauma, genetics and obesity [58]. Without apparent solution, it is a challenge to improve the quality of life when you have this disease. Hence, it is important to know the fundamental risk factors to keep them under control and delay or prevent the occurrence of $\mathrm{OA}$ as much as possible.

\section{$\square$ Age:}

Age is the risk factor with the highest incidence for the development of OA. The time during which a joint is under stress is not the only cause that leads to OA, but a large number of age-related influences[76]. Biomechanics can be altered due to muscular atrophy and the redistribution of forces that accompany it leads to the degradation of the cartilage. The biochemical changes that occur throughout the body with the advance of age is one of the factors that most alter the structure and composition of cartilage [58, 64, 76].

On the other hand, because of the increase in calcification and crystal formation within the joint tissues that occurs with increasing age, the association of calcium pyrophosphate with the presence of osteoarthritis has been well established by radiographic techniques $[77,78]$.

\section{$\square$ Genetic factors}

The genetic contribution to the development of OA has been estimated to range between $40 \%$ and $80 \%$, and a part of the incidence of early OA shows Mendelian inheritance [79]. Evaluating genetic contribution to common OA has led to the identification of 17 loci [80]. The combination of environmental factors with genetic predispositions probably contributes to OA development. Hereditary contributions associated with the development of OA include the bone shape, the superficial area and thickness of the cartilage [58, 79]. Other molecular mechanisms associated with the disease are still under study, like the role of epigenetics, such as DNA-methylation and the role of noncoding RNAs [58, 79, 80]. The advance in knowledge to better understand these processes will allow the development of new therapies directed by genes $[57,58,64,78$ 80].

\section{$\underline{\text { Sex }}$}

Women have a higher prevalence and severity of OA compared to men, with a higher incidence in women who are in the postmenopausal period $[78,81]$. Due to this difference in gender and the accelerated onset of the disease in postmenopausal women, it has been considered that estrogen plays an important role in the development of OA; however, the results provided by clinical trials are contradictory [58, 81]. Anatomical differences have also been observed between men and women. In many cases women have a thinner base cartilage. An additional risk factor is the jarring number of anterior cruciate ligament (ACL) injuries suffered by women compared to men $[58,63,78,82,83]$.

$\square$ Physical activity and occupation

Although practicing sports and maintaining a constant physical activity is beneficial in health issues, doing it inappropriately can become harmful. In the case of joints, the strengthening of the periarticular muscles to help stabilize the joint is of great benefit to the joint as long as it does not have an undue burden on it implying a detrimental effect [64]. Doing strong sports usually affects joint many times in the long term. In a study conducted with soccer players, approximately $80 \%$ of those who had suffered an ACL injury showed radiographic evidence of OA 15 years later [82]. The occupation performed by an individual is also a risk factor since there are occupations in which the workers are exposed to repetitive joint stress. For example, carpenters and dockworkers experience knee OA more frequently; a professional athlete experiences more specific degeneration of the joint than their recreational counterparts [58, 64].

Each joint has its characteristics and its repetitive use when doing a job has been associated with an increased risk of OA. Individuals whose occupations demand them to squat or kneel have double risk of developing the disease; it has greater incidence in individuals who also have to carry or lift weights and in those who have an overweight [64]. The appearance of hip OA has also been associated with prolonged 
positioning and lifting [84]. On the other hand, hand OA manifests more in people who do occupations that require more manual dexterity [85].

\section{$\square$ Obesity}

Among all the risk factors, obesity represents one of the most frequent and important in the OA of peripheral joints, such as the knee and the hip. In conjunction with age, obesity is one of the risk factors for the greatest increase globally, which is why it is expected that knee OA will affect more people in the future. A recent meta-analysis found that a dose-response relationship exists between obesity and the risk of knee OA [86]. The relationship between body weight and hip OA, however, is inconsistent and weaker than that for knee OA [83].

\section{Other factors}

There are many evidences that the biochemistry (cells and chemical components) of the human body plays a fundamental role in the progression of OA. In fact, it can influence in a localized way causing the advancement of the disease either in different joints according to the concentrations of substances in the biological fluids that have contact with these joints $[61,64,87]$. The reactive oxygen species that play a fundamental role in the development of oxidative stress in the body can accumulate in the joints with age and cause cartilage damage. When vitamin $\mathrm{D}$ is low in the body it can increase the incidence and progression of osteoarthritis of the knee and hip [78].

Finally, we can conclude at this point that the risk factors are diverse, each with an incidence in the different joints of the body and that in most cases the development of the disease is conditioned by more than one factor. It should be noted that age is possibly the most important factor since, due to dissimilar causes, the number of people in the older age groups is increasing. On the one hand, the development of science and medicine has allowed the reduction of mortality due to infectious and viral transmission diseases, with which people reach more advanced ages. Bearing in mind that musculoskeletal disorders are the most frequent causes of physical disability, at least in developed countries and that the prevalence of many musculoskeletal disorders increases with age, the likely result is that there will be an increase in the number of people with disorders of chronic incapacity $[62,70]$.

\section{CURRENT TREATMENT METHODS FOR CARTILAGE INJURIES}

The current reality states that the cure of OA is not only a chimera but one of the greatest challenges of humanity and the sciences in general. The increase in life expectancy and the natural tendency of human beings to risk as part of its nature will continue to affect the prevalence and incidence of this disease in the coming years. Despite the expanding comprehension of OA pathogenesis, available therapies are focused on symptom control and, if severe enough, joint replacement. In an age of biologics and small molecule inhibitors for the management of other rheumatic diseases, osteoarthritis sufferers are still limited in their choice of treatments, and the available options are not disease modifying [58].

\section{Treatments for cartilage lesions}

There are currently many palliative treatments for osteoarthritis, but modern science does not rest trying to find the definitive cure, or to improve the effectiveness of existing treatments. Among the most widespread techniques and procedures for the repair or regeneration of articular cartilage are microfracture, mosaicplasty, autologous chondrocyte implantation (ACI) and the matrix-induced autologous chondrocyte implants (MACI).

\section{Microfracture}

In the microfracture procedure, developed in 1997 by Steadman, exposed subchondral bone undergoes small microfractures to achieve localized bleeding that improves chondral renewal [7, 88]. The clinical results of this repair technique, which also introduces cells into the joint, are often unpredictable since the 
new tissue that forms can be a fibrocartilage tissue that degenerates over time $[52,89]$. The microfracture technique could be said to be like a combination therapy which takes advantage of the natural condition of migration of pluripotent progenitor cells to damaged areas with the fact that the damages caused manually by microfracture $[56,90]$.

The clinical results that are achieved with microfracture will depend, among other factors, on the size of the defect and the age of the patient. According to a long-term study in patients with deep subchondral defects, microfracture therapy produced pain relief and restored knee function in $75 \%$ of treated individuals [91]. Despite the high percentage of satisfactory results that are achieved in the initial time after the procedure, it has been shown that after approximately 2 years the clinical results begin to decrease mainly in elderly patients [92]. The major drawbacks of microfracture include the poor biomechanical nature of the repair tissue, partial defect filling, and abnormal bone growth in the lesion area [91, 92].

\section{Mosaicplasty}

The mosaicplasty technique was described in 1993 by Matsusue and is a procedure that is performed in one stage but consists of three steps. It is an arthroscopic procedure indicated on focal and osteochondral focal defects of small and medium size of the femoral condyle. Although the long-term effects have not been thoroughly evaluated, it has been shown that the graft material remains for a short time and that the clinical result shows pain relief and improvement of joint function [94].. Like all surgical procedures, mosaicplasty has some drawbacks: first, disability can occur as a result of removal of the graft in the joint despite the fact that the donor site has no support surface (i.e., donor site morbidity); second, the orientation of the graft in the receptor site, necessary to restore the contour of the femoral condyle is not easily achieved; third, the marginal death of chondrocytes can lead to graft degeneration and, subsequently, to graft failure; finally, there is a risk that the cartilage and bone collapse [7, 52, 93, 94]..

\section{Autologous chondrocyte implantation (ACI)}

The autologous chondrocyte implantation is a first generation cell therapy that constitutes an alternative to treat symptomatic osteochondral defects using a combination of a periosteal patch with suspended autologous cultured chondrocytes. Specifically, the ACI technique consists of seeding autologous chondrocytes in ex vivo scaffolds prior to their placement in vivo in the damaged area. More than 30 years ago, the first patient received an implant with cultured autologous chondrocytes and since then the new cellular therapies that have arisen with the aim of repairing the cartilage are not similar [95, 96].

Like the therapies mentioned earlier in this document, ACI is used to relieve pain and restore the function of knees affected by chondral or osteochondral defects using a two-staged cell-based procedure [97]. The injection of the chondrocytes into the defective area should be aid cellular adhesion and fills in the defect and in theory [93], could produce hyaline-like cartilage rather than fibrocartilage, with subsequent improvement in clinical outcomes $[92,93]$. Due to the repeated and recognized lack of nerves and nutrients in the cartilage, this is one of the most used and successful techniques of all the variants used in modern surgery.

\section{Matrix-induced autologous chondrocyte implantation (MACI)}

Matrix-induced autologous chondrocyte implants is one of the so-called third generation cell therapies developed from the demand for transartroscopic procedures, which is based on the seeding of autologous cultured chondrocytes in a type I/III collagen membrane manufactured from porcine peritoneal tissue, before implantation in a condral defect. These membranes are engineered to promote chondrocyte infiltration on one hand and low friction on the other hand. It is a technique similar to ACI that requires two surgical procedures and the clinical results show that it is possible to repair the articular cartilage. Although MACI is technically attractive in terms of reproducibility, safety, surgical simplicity and reducing invasiveness and intraoperative time, the expense of the technique warrants further investigation [95, 98-100]. 
MACI is an evolution of Carticel (autologous cultured chondrocytes, Genzyme Biosurgery), the only cell therapy for cartilage repair currently available in the United States. Furthermore, the MACI procedure has been widely adopted in routine orthopaedic practice, with the most clinical experience outside of the United States (more than 10.000 implants since the end of the last millennium); the majority of the clinical, histologic, arthroscopic, and magnetic resonance imaging (MRI) data are published from studies conducted in Europe and Australia. Finally, the MACI technique is the only third generation cell carrier that is currently being evaluated in a randomized, controlled trial to meet European regulations for marketing approval and, potentially, those of other countries [95, 99].

\section{BIOMATERIALS FOR CARTILAGE TISSUE ENGINEERING}

From the beginning, biomaterials have been conceptual, theoretical and practically the best solutions that science has found to repair or restore any malfunction of the human organism. But precisely because the cartilaginous tissue is one of the most complex to achieve success in these tasks, due to its mentioned avascularity, aneurality, small amount of cells and lack of nutrients, it has become a really "double" challenge. It must remember that biomaterials are a science of commitment. When you want to maximize a property, it is done regularly sacrificing the values of other. Then, the application becomes a determinant to choose properly what property must be improved.

Throughout history a biomaterial has been described by several authors as 'any material used to manufacture devices to replace a part or function of the body in a safe, reliable, economically and physiologically acceptable way' or 'a synthetic material used to replace part of a living system or to function in intimate contact with living tissue' [101]. More recently, a formal definition established by the Clemson University Advisory Board for Biomaterials is: "a biomaterial is a systemically and pharmacologically inert substance designed for implantation within or incorporation with living systems." These descriptions add to the many ways of looking at the same concept but expressing it in different ways. The variety of devices and materials that are currently used as biomaterials for the treatment of diseases and injuries is very extensive, among the most common we have sutures, dental fillings, catheters, coronary stents, bone plates, etc. It is important to emphasize that the term 'biomaterial' should not be confused with biological materials which are materials like bone, skin or artery produced by a biological system [102].

Because the ultimate goal of using biomaterials is to improve human health by restoring the function of natural living tissues and organs in the body, it is essential to understand the relationships among the properties, functions, and structures of biological materials. Thus, three aspects of study on the subject of biomaterials can be envisioned: biological materials, implant materials, and interaction between the two in the body [101, 102].

\section{Nanobiomaterials for cartilage tissue engineering}

Research in the field of nanobiomaterials has gained great interest in dissimilar applications; its potential to modulate cellular behavior has led to a vertiginous increase in research in the orthopedic domain [103]. Tissue engineering needs an interdisciplinary knowledge base, since it is required, on the one hand, to understand the processes of cell proliferation and differentiation and also to know about the science of substrate materials or the scaffolding materials used in tissue culture or organs [101].

The objective pursued by tissue engineering is the development of substitutes for functional tissues with which damaged organs and tissues can be reconstructed. In the design of a tissue construction, a biomaterial whose characteristics simulate those of an EMC is used and, in addition, cells that improve tissue development are generally seeded. Recently, it has been recognized that nanobiomaterials play a central role in tissue engineering, as they can better support tissue regeneration [104].

In the remainder of this review, the authors want to give a broad view on the possible solutions based on the engineering of cartilage tissue to treat $\mathrm{OA}$; its fundamental objective is targeting polymeric 
nanobiomaterials as a platform to deliver supporting cells, nutrients, and growth factors among others important and possible components to restore the articular cartilage.

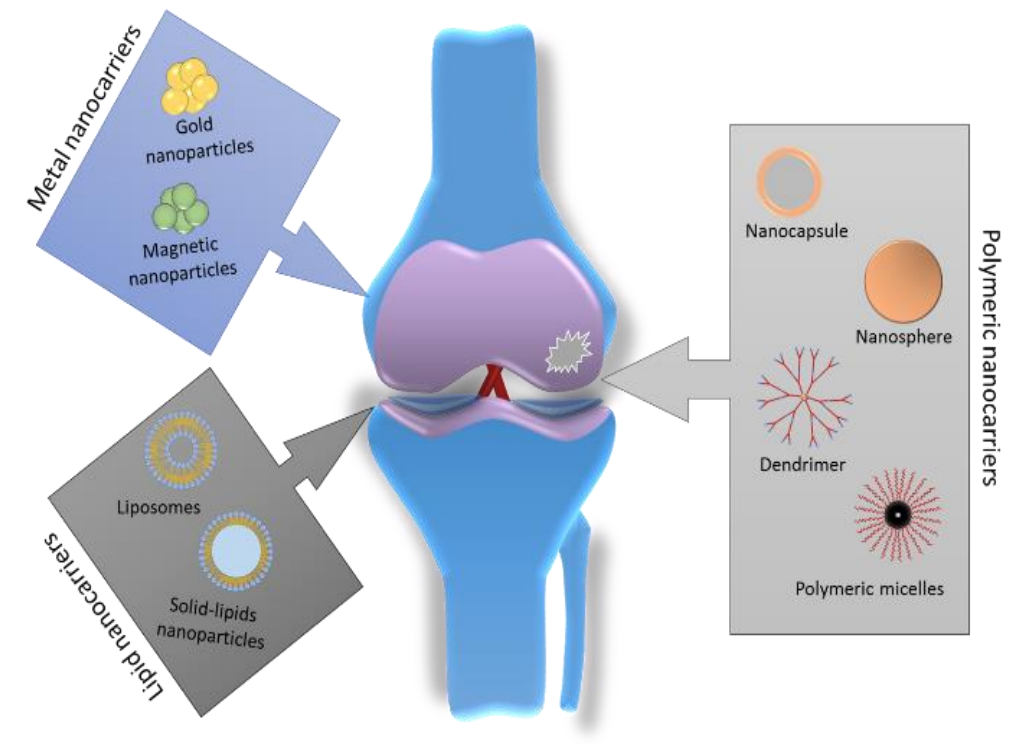

\section{Fig. (2). Different kind of nanocarriers for drug delivery/tissue engineering in osteoarthritis treatment}

\section{Nanoparticles}

Cartilage homeostasis is altered during arthritis due to the inflammatory processes that occur in the joint tissues because of the damage [105]. It has been reported that the diseased chondrocytes that are found inside the cartilage produce inflammatory cytokines which have an important role in the progression of OA $[105,106]$. Therefore, to achieve an effective treatment it is necessary that the therapeutic molecules reach those damaged chondrocytes located inside the cartilage [107]. Currently, for the treatment of OA, various steroidal and non-steroidal anti-inflammatory drugs are used, whose size vary from $0.5 \mathrm{~nm}$ up to $6 \mathrm{~nm}$ in size. It is of interest to develop larger systems for new therapies [108, 109]. Some arthritic diseases such as rheumatoid arthritis have been treated effectively with large therapeutic antibodies, but these antibodies have not yet been evaluated in the treatment of OA [110]. Because of the wide variation in size of potential therapeutics $(0.5 \mathrm{~nm}<$ hydrodynamic radii $<6 \mathrm{~nm})$, understanding how molecular transport occurs within highly heterogeneous cartilage is important to the design of future therapies [111].

The scientific literature of the last decades describes widely the properties of nanoparticles (NPs). Based on this, there are different parameters that can be manipulated to improve the efficiency of carriers, such as chemical composition, surface modification and the use of director ligands, physical properties among others [14]. The properties of the nanoscale are very specific to its inherent condition of small size, for example, the surface area to volume ratio increases, which implies that the reactivity of the surface increases as well; the load capacity of the drug, bioavailability and the release of charged bioactive agents also increase. The small size affects the transport properties since they are structures that can penetrate the tissues through the capillaries and the epithelial lining increasing the diffusivity and biodistribution, with which the targeting capacity of the therapeutic agents towards the target cells is more efficient [112].

The nanoparticles used in the administration of drugs for therapy or for diagnosis are colloidal solids that vary in size from $2 \mathrm{~nm}$ to $1000 \mathrm{~nm}$. However, particles larger than $200 \mathrm{~nm}$ are not widely used because they can activate the immune system. The materials and technologies that have been used for the construction of drug releasing nanosystems are very diverse, but they can be classified in a very general way in 2 large groups: a) Organic nanostructures, group which are the polymeric materials where the nanospheres, nanocapsules, micelles, liposomes, dendrimers and polymer-drug conjugates are constructed; b) Inorganic 
nanostructures in which metal oxide nanoparticles, mesoporous silica nanoparticles and carbon nanotubes are grouped $[113,114]$.

Nanomaterials are used for therapy of diseases through several ways such as targeted drug/gene delivery approaches and induction of damaged tissues regeneration. For targeted drug delivery, it can be used as targeting molecules, carrier systems, or the bioactive drug itself. On the other hand, nanomaterials research for drug delivery has focused mostly on developing carrier systems such as liposomes, polymeric nanoparticles, or metal - based nanoparticles. Although small - molecule drugs are the most commonly used therapeutics for drug delivery approaches, there have been serious advances in producing tailored nanomaterial drugs, mostly in the form of small peptides or their conjugates [115].

The highly hydrated condition of hydrogels and their poor mechanical properties are challenges for in vitro and in vivo studies. To overcome these limitations, combinations of both materials are a highly attractive approach [116]. The application of hydrogel may be better if a nanomaterial is inserted in the hydrogel's matrix. Therefore, current studies have led to the development of nanocomposite hydrogels [117]. Nanoparticles, containing organic/polymeric and inorganic such as hydroxyapatite, clay, graphene and metallic nanoparticles can be used as fillers to reinforce the hydrogel matrix [118]. Indeed, nanoparticles have higher surface area to volume ratios, which increase the surface reactivity, the release of loaded bioactive agents, bioavailability and have superior mechanical properties. Also due to their ability to penetrate into tissues through capillaries and epithelial lining, they improve transport properties and a more efficient delivery of therapeutic agents to target cells [119-121]. Nanoparticles in comparison to microparticles have many advantages. For example, when particles are embedded into the polymeric matrix during the crosslinking of scaffold, they have more homogeneous distribution and much more particles are available for the same equivalent weight of carriers [122, 123].

The drug carriers comprise soluble synthetic polymers, biodegradable liposomes, micro/nanoparticle, nanofibers, dendrimers, and micelles. Nanoparticles derived from poly(lactic-co-glycolic) acid (PLGA), polyglycolic acid (PGA), and polylactic acid (PLA) are commonly applied biomaterials for delivering osteoinductive factors, plasmid DNA (for gene therapy), or anti-inflammatory drugs to enhance bone tissue regeneration. Even some hydrophobic polymers normally notused for their low interaction with fluids, which greatly hinder the possible release of active or biological ingredients, have been used in this function such as poly(tetraethylene glycol methacrylate) or poly(methyl methacrylate). The size of a molecule plays a significant role in effective drug delivery to the target site $[12,14,112,124-130]$.

Altering the surface topography of implants at the nanoscale is another way that current materials can be modified [56]. Nanoparticles, nanodots, or even nanoscale grooves have been engineered onto scaffolds using nanolithography to promote cell attachment to scaffold matrices [131]. Similarly, cell-adhesive peptides can be bound to surfaces at the nanoscale to regulate chondrogenesis and selectively control cellmatrix interactions [132]. Top-down manufacturing techniques such as nanolithography, and bottom-up techniques such as electrospinning represent the latest advances in nanoengineering, making development of novel constructs increasingly feasible [133-135].

\section{Nanoparticles as drug delivery systems for pharmacological OA treatment}

As mentioned earlier, osteoarthritis is characterized, among other factors, by severe pains during movement that decreases at rest. The first line of pharmacological treatment is associated with the reduction of pain, primarily anti-inflammatory drugs and oral steroids are used but they have severe side effects on the gastrointestinal tract [136-138]. In order to eliminate the mentioned problem and to obtain better pharmacological results, the intraarticular injections (IA) are an attractive modality as the drug can be directly given to the main site where the disease has developed. This route of drug administration requires lower doses and reduces the drug distribution to undamaged sites; it allows reaching high concentrations of the drug at the site of action with a lower systemic toxicity. In addition, it allows the administration of proteins and drugs with low solubility that have poor oral bioavailability [139]. 
Non-steroidal anti-inflammatory (NSAID) and corticosteroid drugs injections allow administration of the drugs directly at the damage site and relieve pain. However, they have a short half-life within the joint because of rapid uptake of the drugs by circulation depending on its molecular size and solubility. NSAIDs drugs such as paracetamol, diclofenac, naproxen or ketoprofen have a residence time in synovial fluid (SF) lower than $5 \mathrm{~h}$. This implies that the patient has to receive repeated doses for their treatment [138-140]. Therefore, the development of novel nanoformulations or hydrogels as IA injection drug delivery systems can be used to achieve greater advantages for reducing the rapid clearance. This can be done by increasing the drug's residence time in the synovial cavity allowing the continuous release and slow absorption of the active substance in the joint [141].

From the investigations done so far, the nanostructures used for the design of intra-articular release systems of anti-inflammatory drugs are diverse. Micelles, polymeric nanoparticles, liposomes and lipid nanoparticles are the largest group developed. Likewise, metallic nanoparticles are gaining ground, mainly gold nanoparticles, often in combination with polymers of natural or synthetic origin. Although we have spoken so far of the pharmacological group of NSAIDs and corticosteroids in the treatment of osteoarthritis, in recent years a new group of bioactive substances called disease-modifying osteoarthritis drugs (DMOADs) have been developed, which inhibit the progression of the disease and can improve the symptoms of osteoarthritis and joint function [136, 142].

As described earlier in this section and for a better visualization of the extensive and growing diversity of research developed in the field of "nanobiomaterials applied as vehicles for intra-articular drug delivery systems, we will present a group of examples representative of them.

Japanese authors obtained DL-lactide/glycolide copolymer (PLGA) nanospheres with different molecular weights and loaded with sodium phosphate betamethasone (BSP) by emulsion solvent diffusion method. The obtained nanospheres reached an average diameter between 300 and $490 \mathrm{~nm}$ and the in vitro release of the BSP was controlled by the molecular weight and the ratio lactic acid / glycolic acid (LA / GA) of the polymers. The nanospheres of PLGAs with higher molecular weights and lactic acid content prolonged the release for more than three weeks. The in vivo study carried out in rabbits with osteoarthritis induced by intraarticular injection of ovalbumin showed that the inflammation in the joint was significantly reduced for a period of 21 days when BSP-loaded nanospheres were administrated. In addition, the serum antibody against ovalbumin exhibited a reduction sustained during the period demonstrating prolonged antiinflammatory efficacy [143].

Saadat et al. encapsulated triamcinolone in polymeric micelles based on phospholipid and hyaluronic acid for intaarticular administration. The synthesized micelles reached an average size of about $180 \mathrm{~nm}$ with a spherical morphology. The histopathological knee study of rats treated with the triamcinolone micelles showed no signs of cysts formation, inflammation or accumulation of macrophages in the treated group in respect to the control group. The in vitro release study showed a sustained release for $72 \mathrm{~h}$. To observe the distribution in the synovial space, micelles marked with Cys 7 were administered and it was observed that they remained in the joint for at least 3 days. On the other hand, the plasma concentration of triamcinolone showed lower levels of drug for the micellar formulation compared to the suspension formulation, which indicates a less exposure of the drug to synovial fluid and consequently a lower clearance rate [144].

Elron-Gross et al. developed liposomal formulations to encapsulate dexamethasone and sodium diclofenac independently and in combination. They used two types of bioadhesive liposomes, one based on hyaluronan and the other on collagen. In all cases, a high encapsulation efficiency of more than $80 \%$ was achieved and it was demonstrated that the encapsulated drugs retained their biological activity, even in combined formulations. The in vivo studies showed that with a single dose of any formulations tested it is possible to reduce the knee inflammation of injected mice compared to the control group. The best results were obtained with the formulation of liposomes with hyaluronan and both anti-inflammatory drugs with which the volume of inflammation was reduced up to $12.9 \%$ [145]. 
In the previous examples, we saw nanoformulations designed to encapsulate conventional antiinflammatory drugs, with which it is possible to counteract two of the most notable clinical manifestations of osteoarthritis, inflammation and pain. However, to delay the progress of the disease, achieve even greater improvement and a restoration of the damaged area, nanoformulations of DMOADs or its combination with conventional anti-inflammatory drugs are a more promising alternative.

Researchers from the University of Geneva developed a nanosystem for osteoarthritis treatment. They prepared and encapsulated kartogenin nanocrystals, a small molecule that induces chondrogenesis, in a PLA matrix marked with cyanine 7 by spray drying. The nanoparticles obtained showed a spherical morphology with a granular surface due to the presence of nanocrystals distributed in the matrix. The in vitro release of kartogenin was monitored for 3 months in saline buffer at $\mathrm{pH} 7.4$; a burst effect was seen in the first $8 \mathrm{~h}$ due to the presence of nanocrystals on the surface of the nanoparticles. The in vivo tests showed a localized retention of the nanoparticles in the joint for 2 months. The quantification of OA biomarkers in plasma from mice on day 56 was evaluated by multiple ELISA. Compared with the KGN solution, treatment with KGNNPP led to a more significant effect on vascular endothelial growth factor (VEGF), which has a key role in the metabolism of chondrocytes and progression of the illness [146].

Another group from the University of Dongguk obtained nano/microparticles of chitosan conjugated with kartogenin for the regeneration of articular cartilage. The ionic gelation method with sodium tripolyphosphate was used for the preparation of the nano/microparticles. The in vitro release of kartogenin was followed for 7 weeks and a sustained and continuous release was observed for both particles. In the cytotoxicity assays both particles showed normal proliferation at concentrations below $100 \mathrm{nM}$ of kartogenin. The retention times of the particles in the joint of rats with osteoarthritis induced were investigated by fluorescence imaging and the signals were observed for 24 days after administration. Histological studies performed with safranin-O staining demonstrated a clear cartilage regeneration in the rats treated with the chitosan-kartogenin particles. The negative control group treated with PBS showed extensive areas of cartilage destruction, with loss of matrix and denudation of the surface while rats treated with free kartogenin showed less cartilage loss with vertical fissures of the matrix. The detection by immunofluorescence of COL2 and aggrecan showed that the particles of chitosan-kartogenin regenerated the articular cartilage and stopped the progression of OA with greater efficacy than kartogenin [147]. In this same research group, they obtained thermosensitive nanospheres of pluronic F127 with chitosan oligosaccharides for the combined release of diclofenac sodium and kartogenin. Sodium diclofenac was encapsulated in the matrix core and kartogenin was covalently crosslinked to the outside of the nanosphere. In this case, the release of both compounds was influenced, among other variables, by temperature due to the properties of pluronic F127. At lower temperatures there was greater swelling which favored penetration and exchange of the surrounding fluid with the drugs in the particle. Likewise, the time of the particles in the joint was observed and it was estimated that the elimination half-life at the injection site was $115.2 \pm$ $2.2 \mathrm{~h}$ for the nanospheres with cold treatment and 109.3 $\pm 3.6 \mathrm{~h}$ for those without treatment [148].

In the pathogenesis of cartilage osteoarthritis, one of the key aspects is related to the degradation produced by the release of proteinases and inflammatory cytokines such as IL- $1 \beta$ and TNF- $\alpha$. These mediators cause a decrease in collagen synthesis and increase the production of degradation proteases, including matrix metalloproteinases (MMPs) [141]. Another of the most novel treatment pathways of osteoarthritis is the administration of inhibiting bioactive molecules of interleukin-1 (IL-1) such as IL-1Ra protein and diacerein or MMP inhibitors such as salmon calcitonin. Some published works showed the use of nanosystems in improving the bioavailability and release of these molecules in the intra-articular region and thus enhancing their pharmacological action.

Although nanoparticles are a promising strategy for intra-articular administration, they do not allow the encapsulation of all types of active substances, such as proteins, which are highly sensitive molecules. Whitmire et al. designed a block copolymer that self-assembles into submicrometer particles and provides protein binding sites to control the release. The IL-1Ra protein used in this study was linked through a 
peptide bond. The polymeric nanoparticles bound to IL-1Ra not only retained the bioactivity of IL-1Ra and its ability to attack synoviocytes, but also modulated the activation of NF- $\kappa B$ after stimulation with IL-1 $\beta$, clearly indicating that IL-1Ra maintains its ability to block IL-1. The ability of nanoparticles to retain IL$1 \mathrm{Ra}$ in the rat joint for 14 days was evaluated and showed that the nanoparticles bound with IL-1Ra significantly increased the retention time of IL-1Ra in the rat joint and maintained the structure and composition of the cartilage [127]. Following this same line of treatment, another study associated with the inhibition of interleukin 1 was developed by Jain et al, in which they obtained solid lipid nanoparticles loaded with diacerein and modified with chondroitin sulfate. The size range in the prepared formulations was $396 \pm 2.7 \mathrm{~nm}$. A prolonged release of up to $16 \mathrm{~h}$ was observed; the value was superior to the result obtained for the release of the dispersed drug, with an increase in the bioavailability of diacerein by 2.8 times. Concentration of rhein in the synovial fluid for different formulations was estimated and was found to be significantly higher for nanoparticles loaded with diacerein and modified with chondroitin sulfate. Also the histological observations resulted in a better efficacy of the drug when it was administered in this formulation [149].

Salmon calcitonin (SC) is an analgesic peptide that has achieved great interest in the treatment of OA due to its beneficial metabolic actions on cartilage, which also blocks the degradation of collagen in chondrocytes exposed to TNF- $\alpha$ and inhibits the expression of MMP. Considering that peptides are molecules with a high sensitivity to proteases action, their encapsulation helps and potentiates their effect once incorporated into the organism. Ryan et al. encapsulated this peptide and hyaluronic acid (HA) in a chitosan (CS) matrix to form a nanocomplex in form of nanoparticles. The aim of this study was to evaluate whether encapsulated SC reduced the levels of MMP and the expression of inflammatory genes, in this case NR4A2 mRNA, previously induced in human chondrocytes for in vitro assays and in addition the behavior in vivo, compared with a control of dexamethasone and PBS. In vitro studies showed that the combined nanoparticles of SC and HA in the CS matrix significantly reduced the expression of NR4A2 and MMP13. In the case of in vivo tests, nanoparticles applied in the murine model also reduced the diameter of the inflamed knee in a similar way with dexamethasone, which demonstrates the potential of nanoformulations for intra-articular treatment of knee OA [141].

Continuing with the same line on the use of anti-inflammatory peptides, we should highlight the research carried out by the group of Alyssa Panitch in which they encapsulated the peptide KAFAK (short name, inhibitor of protein kinase 2) in two matrices based on poly(N-isopropylacrylamide) and 2-acrylamido-2methyl-1-propanesulfonic acid (AMPS). In a first study carried out in 2013, they encapsulated the peptide in the nanoparticles by standard precipitation polymerization. Subsequently, in 2016 they incorporated polyethylene glycol (PEG) into the formulation to reduce the binding to non-specific proteins. In both cases, the results were satisfactory demonstrating the ability of the nanoparticles to selectively release the encapsulated peptide and suppress cytokine production significantly. However, nanoparticles with PEG showed greater stability and retention in the joint and released less than $10 \%$ loaded KAFAK for $96 \mathrm{~h}$ at $\mathrm{pH}$ $7.4[150,151]$.

Similarly, to improve the retention of the nanoparticles in the synovial cavity, cationic polymers that interact ionically with the hyaluronic acid present in the extracellular matrix of the cartilage and in the synovial fluid can be used. Morgen et al. made a detailed study of different formulations of core-shell type nanoparticles composed of a core of neutral polymer with a cationic polymer shell. A fluorescent peptide (RFK peptide labeled with fluorescein isothiocyanate) was bound to the core-forming polymer to evaluate its release in vitro and in vivo. According to the in vitro results the release of the conjugated peptide was $20 \%$ in 6 days which provides a duration of about one month of exposure of a therapeutic agent after the injection. Similarly in the in vivo results a loss of fluorescence in the knee of approximately $25 \%$ in a week was observed which is very similar to the result in vitro [142].

\section{Gene therapy and nanoparticles for $O A$ treatment}


In recent years gene therapy has been widely investigated for the treatment of OA and has proved to be a promising alternative because it allows treating the causes instead of the symptoms [152]. Viral and nonviral systems can be used, but viral systems, despite their high transfection efficiency and which allow the protection of deoxyribonucleic acid (DNA) against enzymatic attack, have the disadvantage of generating an undesired immune response [153]. Then, non-viral administration systems are viable candidates for the treatment of osteoarthritis. Non-viral delivery vectors can be peptides, polymers, liposomes or dendrimers that penetrate cells (polymers with multiple branches).

The biological factors that regulate cartilage degeneration in OA are diverse, including: NF- $\kappa \mathrm{B}, \mathrm{HIF}-2 \alpha$, interleukin-1 beta (IL-1 $\beta$ ), tumor necrosis factor- $\alpha$ (TNF- $\alpha$ ), matrix metalloproteinases (MMP) and disintegrin and metalloproteinase with thrombospondin motifs (ADAMTS) [153-155]. These factors are an important group of therapeutic targets for the action of vectors in gene therapy for the treatment of OA. In the same way that it has been described throughout this work for non-virus-mediated gene therapy, it is also necessary to ensure that target genes are targeted directly to chondrocytes and thus increase efficiency and specificity [156]. Again the design of nanostructured matrices provides a solution to the existing needs.

Sun Pack et al. obtained nanoparticles of PLGA loaded with dexamethasone which was subsequently complexed with poly(ethylenimine) (PEI)/siRNA; to observe co-administration the cells were transfected with fluorescent protein siRNA (GFP-siRNA) and the drug. After transfection with GFP siRNA, 70\% reduction of C28/I2 cells demonstrated GFP expression, while MOCK that transported PLGA nanoparticles and PLGA nanoparticles without siRNA showed no differences in GFP expressions. The combined treatment with dexamethasone and COX-2 siRNA clearly reduced the expression of inflammatory and apoptosis-related factors produced in C28 / I 2 cells, which were induced to enter an inflammatory state by TNF- $\alpha(10 \mathrm{ng} / \mathrm{mL})$. However, soluble dexamethasone alone had little effect in reducing the production of inflammatory factors and related to apoptosis [155].

Lu et al. prepared chitosan-graft-polyethylenimine (CP) / DNA nanoparticles as novel non-viral gene vectors for gene therapy of osteoarthritis. The size and $\mathrm{z}$ potential of the nanoparticles depended on the weight ratio of $\mathrm{CP}$ : DNA, where at a higher $\mathrm{CP}$ content the particle size decreased and the surface charge increased. The transfection efficiency of the CP / DNA nanoparticles was dependent on the CP: DNA ratio (w/w) and was markedly higher than that of the nanoparticles from polymers separately and that of the naked pDNA, behaving similarly to the product Lipofectamine TM 2000. The cell viability of the chondrocytes and synoviocytes tested was $>90 \%$ in the CP / DNA nanoparticles. This viability was much greater than that of the PEI $(25 \mathrm{kDa}) / \mathrm{DNA}$ nanoparticles. Intracellular traffic studies found that $\mathrm{CP}$ copolymers were able to efficiently transport the pDNA within the chondrocytes and synoviocytes, and the pDNA could be detected upon entering the nucleus after $4 \mathrm{~h}$ of incubation [152]

\section{Nanohydrogels}

The concept of nanohydrogels can be confusing, starting from the true concept of hydrogel. What is really called' nano'? When it concerns nanohydrogels, does it refer to their particles? But hydrogels can be manufactured in different shapes, for example three-dimensional scaffolds. Then, what is 'nano' and what is the size of its pores?

From the point of view of the publications reviewed, the two most accepted concepts for this type of classification relate precisely to the size of hydrogel particles and pores. All the above mentioned is very related to the manufacturing of the material and the ability to exchange (diffusion) liquids with the surrounding environment that has the manufactured structure.

The interactions between the materials and the active molecules as well as their characteristics condition the speed and distance that molecules will diffuse. The nanostructure of the gels depends on different factors such as the polymer fraction used, the type of polymer, its molecular weight, the size, the concentration of the crosslinking agent that is used $[157,158]$. 
In a study developed by Lim et al, bone morphogenetic proteins (BMP-2) were encapsulated in negatively charged alginate nanohydrogels using the reverse emulsification and evaporation method with an encapsulation efficiency of $67 \%$. The encapsulated proteins were released within 7 days with an initial release of $40 \%$ in $24 \mathrm{~h}$. It was further confirmed that the BMP-2 released helped in the differentiation of human bone marrow stromal cells (bMSCs) in osteoblasts [159, 160].

Research about a new type of redox/pH dual stimuli-responsive poly(methacrylic acid)-based nanohydrogels from methacrylic acid and N,N-bis(acryloyl)cystamine crosslinker via distillationprecipitation polymerization has been developed. The nanohydrogels could be easily degraded into individual linear short chains in the presence of $10 \mathrm{mM}$ dithiothreitol (DTT) or glutathione (GSH). Doxorubicin (DOX) as a model anti-cancer drug was highly and efficiently loaded into these nanohydrogels (up to $42.3 \mathrm{wt} \%$ ) due to the strong electrostatic interactions between the amine group in DOX and the carboxyl groups in the nanohydrogels at a physiological $\mathrm{pH}$. The drug release behavior of the DOX-loaded nanohydrogels in the presence of GSH was very different from the DTT as the loaded DOX could be quickly released in the presence of GSH, but not in DTT. The possible reason is the synergic effect of reduction and charge exchange of GSH at a low pH. Finally, these nanohydrogels had excellent colloidal stability and could effectively entrap DOX (anticancer drugs); the highest loading efficiency of DOX could approach 95.7\%. The disulfide linkage provides the nanohydrogel redox responsive capability, and could degrade the nanohydrogels into short polymer chains using water-soluble reducing agents like dithiothreitol (DTT) and glutathione (GSH). The DOX release data demonstrated that the degradation-triggered drug release was very different in the presence of GSH and DTT as well as at different $\mathrm{pH}$. The nanohydrogels were nontoxic to normal HEK 293 cells and the DOX-loaded nanohydrogels possessed excellent killing effect on glioma tumor cells, suggesting that they may be effective in delivering anti-cancer drugs to tumors. [161]. In this study, the researchers used DOX as a model drug to demonstrate that the nanohydrogels developed are dual stimuli-responsive systems. Considering the aim of this review, the system developed by the authors could have appropriate characteristics to be used in the release of steroids anti-inflammatory drugs for example, and allow their use in the treatment of damaged cartilages by intra-articular application.

On the other hand, thermally responsive poly( $\mathrm{N}$-isopropylacrylamide-co-acrylamide) nanohydrogels were synthesized in this study by free-radical precipitation polymerization method. Different lower critical solution temperatures (LCST) of nanohydrogels were obtained by modulating the amount of acrylamide and characterized by measuring their transmittances of the particle solutions at $500 \mathrm{~nm}$. The diameters within the range of 50-450 $\mathrm{nm}$ were achieved by manipulating the amount of sodiumdodecyl sulfate (SDS). Near infrared dye NIRD-12, with excitation and emission wavelengths at $772 \mathrm{~nm}$ and $814 \mathrm{~nm}$, was entrapped into the copolymeric nanohydrogels for in vivo animal studies. The various thermally targeted tests indicated that the thermally responsive nanohydrogel can only accumulate in tissue with elevated temperature, both in normal tissue and tumor. The targeted behavior of this kind of thermally responsive nanohydrogel is passive and non-specific. The targeted locations can be selected by manipulating the suitable LCST of nanohydrogel and tissue heating process. Results indicated that the thermal responsive nanohydrogels in combination with local hyperthermia treatment provided a promising way for tumor therapy. Entrapment of anticancer drugs into this kind of nanohydrogels for tumor therapy will be our future work [162].

Other researchers report the synthesis and characterization of size-controllable and stimuli-responsive DNA nanohydrogels as effective targeted gene delivery vectors. DNA nanohydrogels were created through a self-assembly process using three kinds of building units, respectively termed Y-shaped monomer A with three sticky ends (YMA), Y-shaped monomer B with one sticky end (YMB) and DNA linker (LK) with two sticky ends. Hybridization at the sticky ends of monomers and LK leads to nanohydrogel formation. DNA nanohydrogels are size-controllable by varying the ratio of YMA to YMB. This study has demonstrated that two kinds of Y-shaped monomers and DNA linker can self-assemble in a one-step reaction to generate DNA nanohydrogels with controllable size. By incorporating different functional elements, such as aptamers, disulfide linkages and therapeutic genes into different building units, the synthesized Y-gel-Apt can be used 
for targeted and stimuli-responsive gene therapy, resulting in the inhibition of cell proliferation and migration in A549 cells. The advantages of these DNA nanohydrogels include easy synthesis without enzymatic ligation or photo-polymerization, controlled size, efficient cellular uptake, enhanced nuclease resistance and superior biocompatibility. Furthermore, these nanohydrogels can be used as safe nanocarriers for the delivery of drug or imaging agents. Thus, the proposed multifunctional aptamer-based DNA nanohydrogels will find potential applications for point-of-care diagnosis, efficient drug transport, and improved targeted OA therapeutics. [163].

\section{Other matrices}

All current strategies and procedures are based on decreasing chronic pain and improving joint function, but all fail in two fundamental areas: restoring the original cartilage structure and reproducing its biomechanical properties.

That is why current research focuses on tissue engineering whose procedures involve possible solutions to the main difficulties. It is good to remember that this is a scientific challenge because restoring or regenerating tissue which in principle does not offer possibilities to do so due to its composition and complexity is still an unsolved problem [7].

The term of the triad of tissue engineering has been so named since in current strategies the combination of three fundamental components is used: the carrier matrix, appropriate cells and bioactive molecules [164, 165]. A cartilage developed by tissue engineering must be structurally similar to the native cartilage; for this it must have biphasic properties, a fluid phase composed of $80 \%$ water and $<1 \%$ electrolyte and another solid phase containing between $10-20 \%$ type II collagen and 5-10\% proteoglycans [166].

The inclusion of chondrocytes in a three-dimensional (3D) support material greatly facilitates the transplant procedure, and this can be carried out minimally invasively by an arthroscopic technique that also does not require the collection of the periosteal flap [164, 167]. Tissue engineering scaffolds should behave similarly to the ECM of native cartilage to allow cell-to-cell proliferation, differentiation, and cell proliferation processes to be carried out properly. The scaffolds of collagen, hyaluronate, chondroitin sulfate and gelatin are the best examples of matrices for this purpose. The scaffolds developed for this application must be biocompatible, allow a uniform cellular distribution, stable in a suitable period of time and favor a good integration with the surrounding native cartilage. The mechanical properties of the scaffold have to ensure that it is stable against the forces of compression and wear that occur in an in vivo joint, and should be remodeled from the gradual replacement by ECM of cartilage-like type secreted from the cells [165]. On the other hand, the proliferation and phenotype of the chondrocytes will depend to a great extent on the nature of the scaffold material and its porosity [168].

During an ACT procedure, the dedifferentiation that is observed of the chondrocytes in the monolayer culture can be avoided by incorporating cells into a 3D support material $[164,167]$. In a three-dimensional alginate scaffold, the typical phenotype of chondrocytes can be maintained for at least 8 months [169]. A scaffold that presents a structure with pore sizes between 250 and $500 \mathrm{~nm}$ favors a better proliferation of chondrocytes and the secretion in ECM [168]. Scaffolding materials utilized for articular cartilage tissue engineering can be subdivided into natural and artificial materials.

Natural materials used in tissue engineering to produce three-dimensional structures for cartilage include agarose, alginate, hyaluronic acid, gelatin, fibrin glue, collagen derivatives and acellular cartilage matrix. However, they present problems that affect their possible application such as less mechanical resistance, disease transfer and antigenicity. They are also prone to rapid and variable degradation [170].

Alginate cell suspensions may be dripped into $\mathrm{CaCl}_{2}$ to form 3D nodules. The cells may be released from the alginate by mild chelation. This scaffold system has been well documented for chondrocyte and chondroinduced stem-cell growth in vitro [171]. Researchers at Wurzburg University, Germany used an ultra-highviscosity/clinical-grade alginate to propagate chondrogenesis in a murine mesenchymal C3H10T1/2 progenitor cell line [172]. 
Another study comparing chondrocyte cells (CC) seeded in 2\% and 3\% agarose showed that, at 28 days of culture in $20 \%(\mathrm{v} / \mathrm{v})$ fetal bovine serum, $2 \%$ agarose constructs were stiffer and had a higher collagen content [173]. Agarose has been useful for investigating the effects of mechanical loading on cartilage formation [174]. Rahfoth and colleagues performed allograft transplants of CC in agarose gel into osteochondral defects in the knees of rabbits. They reported no graft $v s$ host rejections or foreign-body immune responses and, after 18 months, $47 \%$ of grafts developed a morphologically stable hyaline cartilage.

In recent years, cross-linked collagen has been popularized because of its improved mechanical characteristics and slower degradation rates. Collagen may be polymerized by cross-linking agents such as epoxy compounds or carbodi-imide groups, and the collagen may be prepared in water or acetic acid [175]. Collagen I sponges seeded with fetal bovine epiphyseal chondrocytes showed comparable chondrogenesis in the native and cross-linked form, with notably lower matrix accumulation of GAGs than that which is found in normal cartilage [176]. When combined with chondroitin sulfate, collagen II appears to promote more diffuse chondrogenesis and matrix production than does collagen I [175].

Mesenchymal progenitor cells (MPCs) can be evenly distributed onto Gelfoam ${ }^{\circledR}$ (a patented gelatin construct manufactured by Pharmacia and Upjohn) and, using a chondrogenic medium containing TGF- $\beta 3$ (transforming growth factor $\beta 3$ ), will produce cartilaginous matrix and maintain chondrocytic morphology [177]. As with cell-pellet culture, a thin fibrous capsule forms around the neocartilage. This may interfere with integration of the implant in vivo. Unseeded Gelfoam ${ }^{\circledR}$ constructs implanted into osteochondral defects in rabbits have demonstrated good biocompatibility. At 4 weeks, post-procedure, these defects were 10-20 $\%$ of their original size, but the predominant repair tissue was bone with some cartilaginous regions. Good biocompatibility was demonstrated by minimal inflammation and giant-cell infiltration in the explant from which the gelatin sponge had completely disintegrated. Quintavalla and co-workers made an autologous implant for osteochondral defects in goats using Gelfoam ${ }^{\circledR}$ and MPCs labeled with a fluorescent dye [178].

Chondrocytes and stem cells suspended in fibrin glue and implanted into a thymic and severe combined immunodeficiency disease mice resulted in the production of hyaline neocartilage [179]. Cartilage formation was reported in $87 \%$ of these specimens, but a parallel study including insulin-like growth factor1 and basic fibroblastic growth factor in the scaffold resulted in minimal cartilage production. Peretti et al. found that when devitalized cartilage was added to a fibrin chondrocyte construct, the mass was maintained and new matrix formation was promoted [180].

\section{CONCLUDING REMARKS}

After the vast literature consulted, we can conclude that the growth in the development and production of nanoparticles worldwide has been exponential, mainly due to their excellent physicochemical properties $[181,182]$. In this era, the inventors, producers and consumers of almost all sectors have the opportunity to link nanotechnology to their work. Nanoparticles have reached applications in almost all products in the market from pharmaceuticals, electronics, cosmetics, fabrics, sports equipment to almost any space. As compared to the bulk material, nanoparticle shows higher surface to volume ratio, results in an enhanced contact area with their surroundings and specially designed nanoparticles can be used for immunomodulatory purposes to serve specific functions like anti-inflammatory, vaccine adjuvant and immunosuppressive drugs [183].

\section{CONFLICT OF INTEREST}

The authors declare no conflict of interest, financial or otherwise.

\section{ACKNOWLEDGEMENTS}

This work is part of the research programme 723.012.110 (Vidi), which is financed by the Netherlands Organisation for Scientific Research (NWO). Also, we would like to thank the financial support of the LUMC fellowship grant, project grants from the EU Program H2020-MSCA-2015-RISE (644373- 
PRISAR) and MSCA-ITN-2015-ETN (675742-ISPIC), H2020-MSCA-2016-RISE (734684-CHARMED) and H2020-MSCA-RISE-2017-CANCER (777682).

\section{REFERENCES}

1. Horvai, A., Anatomy and Histology of Cartilage, in Cartilage Imaging. Significance, Techniques and New Developments, T.M. Link, Editor. 2011, Springer: New York. p. 1-10.

2. Meyer, U. and H.P. Weissman, Bone and Cartilage Engineering. 2006, Berlin Heidelberg: Springer Verlag.

3. Hall, B.K., Bones and Cartilage. Developmental and Evolutionary Skeletal Biology. 2015, London: Academic Press, Elsevier.

4. Vunjak-Novakovic, G., et al., Dynamic Cell Seeding of Polymer Scaffolds for Cartilage Tissue Engineering. Biotechnology Progress, 1998. 14(2): p. 193-202.

5. Wong, M. and D. Carter, Articular cartilage functional histomorphology and mechanobiology: a research perspective. Bone, 2003. 33(1): p. 1-13.

6. Vega, S.L., M.Y. Kwon, and J.A. Burdick, Recent advances in hydrogels for cartilage tissue engineering. European Cells \& Materials, 2017. 33: p. 59-75.

7. Ahmed, T.A.E. and M.T. Hincke, Strategies for articular cartilage lesion repair and functional restoration. Tissue Engineering: Part B, 2010. 16(3): p. 305-329.

8. Periyasamy, P.C., et al., Nanomaterials for the Local and Targeted Delivery of Osteoarthritis Drugs. Journal of Nanomaterials, 2012. 2012: p. 13.

9. Vinatier, C. and J. Guicheux, Cartilage tissue engineering: From biomaterials and stem cells to osteoarthritis treatments. Annals of Physical and Rehabilitation Medicine, 2016. 59(3): p. 139-144.

10. Liu, Y., G. Zhou, and Y. Cao, Recent Progress in Cartilage Tissue Engineering-Our Experience and Future Directions. Engineering, 2017. 3(1): p. 28-35.

11. Chuah, Y.J., et al., Hydrogel based cartilaginous tissue regeneration: recent insights and technologies. Biomaterials Science, 2017. 5(4): p. 613-631.

12. Monteiro, N., et al., Nanoparticle-based bioactive agent release systems for bone and cartilage tissue engineering. Regenerative Therapy, 2015. 1: p. 109-118.

13. Hasan, A., et al., Nanoparticles in tissue engineering: applications, challenges and prospects. International journal of nanomedicine, 2018. 13: p. 5637-5655.

14. Santo, V.E., et al., From nano- to macro-scale: nanotechnology approaches for spatially controlled delivery of bioactive factors for bone and cartilage engineering. Nanomedicine, 2012. 7(7): p. 10451066.

15. Koo, O.M., I. Rubinstein, and H. Onyuksel, Role of nanotechnology in targeted drug delivery and imaging: a concise review. Nanomedicine: Nanotechnology, Biology and Medicine, 2005. 1(3): p. 193212.

16. Walsh, W.R., et al., Cell Structure and Biology of Bone and Cartilage, in Handbook of Histology Methods for Bone and Cartilage, Y.H. An and K.L. Martin, Editors. 2003, Humana Press Inc: Totowa, New Jersey. p. 36-58.

17. Torzilli, P.A., Water content and equilibrium water partition in immature cartilage. Journal of Orthopaedic Research, 1988. 6(5): p. 766-769.

18. Maroudas, A., Balance between swelling pressure and collagen tension in normal and degenerate cartilage. Nature, 1976. 260(5554): p. 808-809.

19. Mow, V.C., A. Ratcliffe, and A. Robin Poole, Cartilage and diarthrodial joints as paradigms for hierarchical materials and structures. Biomaterials, 1992. 13(2): p. 67-97.

20. Broom, N.D. and H. Silyn-Roberts, The Three-Dimensional 'Knit' of Collagen Fibrils in Articular Cartilage. Connective Tissue Research, 1989. 23(4): p. 261-277.

21. Chen, M.-H. and N. Broom, On the ultrastructure of softened cartilage: a possible model for structural transformation. Journal of Anatomy, 1998. 192(3): p. 329-341. 
22. Diab, M., J.-J. Wu, and D.R. Eyre, Collagen type IX from human cartilage: a structural profile of intermolecular cross-linking sites. Biochemical Journal, 1996. 314(1): p. 327-332.

23. Eyre, D.R., The collagens of articular cartilage. Seminars in Arthritis and Rheumatism, 1991. 21(3, Supplement 2): p. 2-11.

24. Wu, J.-J. and D.R. Eyre, Covalent Interactions of Type IX Collagen in Cartilage. Connective Tissue Research, 1989. 20(1-4): p. 241-245.

25. Wu, J.-J. and D.R. Eyre, Structural analysis of cross-linking domains in cartilage type XI collagen Insights on polymeric assembly. Journal of Biological Chemistry, 1995. 270(32): p. 18865-18870.

26. Eyre, D., Articular cartilage and changes in Arthritis: Collagen of articular cartilage. Arthritis Research \& Therapy, 2001. 4(1): p. 30-35.

27. Wu, J.J., P.E. Woods, and D.R. Eyre, Identification of cross-linking sites in bovine cartilage type IX collagen reveals an antiparallel type II-type IX molecular relationship and type IX to type IX bonding. Journal of Biological Chemistry, 1992. 267(32): p. 23007-14.

28. Loeser, R.F., et al., Integrin expression by primary and immortalized human chondrocytes: evidence of a differential role for $\alpha 1 \beta 1$ and $\alpha 2 \beta 1$ integrins in mediating chondrocyte adhesion to types II and VI collagen. Osteoarthritis and Cartilage, 2000. 8(2): p. 96-105.

29. Kuettner, K.E., Biochemistry of articular cartilage in health and disease. Clinical Biochemistry, 1992. 25(3): p. 155-163.

30. Hambach, L., et al., Severe disturbance of the distribution and expression of type VI collagen chains in osteoarthritic articular cartilage. Arthritis \& Rheumatism, 1998. 41(6): p. 986-996.

31. Fell, H.B., The histogenesis of cartilage and bone in the long bones of the embryonic fowl. Journal of Morphology, 1925. 40(3): p. 417-459.

32. Randolph, M.A., K. Anseth, and M.J. Yaremchuk, Tissue engineering of cartilage. Clinics in Plastic Surgery, 2003. 30(4): p. 519-537.

33. Bullough, P., Joints, in Histology for pathologists, S. Mills, Editor. 2007, Lippincott Williams \& Wilkins: Philadelphia. p. 97-122.

34. Barnett, C.H. and O.J. Lewis, The evolution of some traction epiphyses in birds and mammals. Journal of Anatomy, 1958. 92(Pt 4): p. 593-601.

35. Poole, C.A., M.H. Flint, and B.W. Beaumont, Morphological and functional interrelationships of articular cartilage matrices. Journal of Anatomy, 1984. 138(Pt 1): p. 113-138.

36. Poole, C.A., S. Ayad, and J.R. Schofield, Chondrons from articular cartilage: I. Immunolocalization of type VI collagen in the pericellular capsule of isolated canine tibial chondrons. Journal of Cell Science, 1988. 90(4): p. 635-643.

37. Bradamante, Z., B. Levak-Svajger, and A. Svajger, Differentiation of the secondary elastic cartilage in the external ear of the rat. International Journal of Developmental Biology, 2002. 35(3): p. 311-320.

38. Eyre, D.R. and H. Muir, The distribution of different molecular species of collagen in fibrous, elastic and hyaline cartilages of the pig. Biochemical Journal, 1975. 151(3): p. 595-602.

39. Knudson, C.B. and W. Knudson, Cartilage proteoglycans. Seminars in Cell \& Developmental Biology, 2001. 12(2): p. 69-78.

40. Hall, A., E. Horwitz, and R. Wilkins, The cellular physiology of articular cartilage. Experimental Physiology, 1996. 81(3): p. 535-545.

41. Loeser, R.F., Chondrocyte integrin expression and function. Biorheology, 2000. 37(1, 2): p. $109-116$.

42. Gelse, K., E. Pöschl, and T. Aigner, Collagens-structure, function, and biosynthesis. Advanced Drug Delivery Reviews, 2003. 55(12): p. 1531-1546.

43. Mendler, M., et al., Cartilage contains mixed fibrils of collagen types II, IX, and XI. The Journal of Cell Biology, 1989. 108(1): p. 191-197.

44. Poole, A.R., et al., Composition and Structure of Articular Cartilage: A Template for Tissue Repair. Clinical Orthopaedics and Related Research, 2001. 391: p. S26-S33.

45. Aigner, T., et al., Independent expression of fibril-forming collagens I, II, and III in chondrocytes of human osteoarthritic cartilage. The Journal of Clinical Investigation, 1993. 91(3): p. 829-837. 
46. Wotton, S.F. and V.C. Duance, Type III collagen in normal human articular cartilage. The Histochemical Journal, 1994. 26(5): p. 412-416.

47. Gomes Jr, R.R., M.C. Farach-Carson, and D.D. Carson, Perlecan functions in chondrogenesis: insights from in vitro and in vivo models. Cells Tissues Organs, 2004. 176(1-3): p. 79-86.

48. Sabatini, M., P. Pastoureau, and F. De Ceuninck, Cartilage and Osteoarthritis. Cellular and molecular tools. Methods in Molecular Medicine, ed. J.M. Walker. Vol. 1. 2004, Totowa, New Jersey: Humana Press Inc. 374.

49. Siódmiak, J., et al., Molecular Dynamic Analysis of Hyaluronic Acid and Phospholipid Interaction in Tribological Surgical Adjuvant Design for Osteoarthritis. Molecules, 2017. 22(9): p. 1436-1456.

50. Levingstone, T.J., et al., A biomimetic multi-layered collagen-based scaffold for osteochondral repair. Acta Biomaterialia, 2014. 10(5): p. 1996-2004.

51. Nguyen, L.H., et al., Engineering articular cartilage with spatially-varying matrix composition and mechanical properties from a single stem cell population using a multi-layered hydrogel. Biomaterials, 2011. 32(29): p. 6946-6952.

52. Becerra, J., et al., Articular cartilage: structure and regeneration. Tissue Engineering. Part B, 2010. 16(6): p. 617-27.

53. Guilak, F., et al., Mechanical and biochemical changes in the superficial zone of articular cartilage in canine experimental osteoarthritis. Journal of Orthopaedic Research, 1994. 12(4): p. 474-484.

54. Buckwalter, J.A. and H.J. Mankin, Articular Cartilage: Part I Tissue Design and Chondrocyte-Matrix Interactions. Journal of Bone and Joint Surgery, 1997. 79(4): p. 600-611.

55. Speer, D.P. and L. Dahners, The collagenous architecture of articular cartilage. Correlation of scanning electron microscopy and polarized light microscopy observations. Clinical orthopaedics and related research, 1979. 139(2): p. 267-275.

56. Kumar, R., M. Griffin, and P.E. Butler, A Review of Current Regenerative Medicine Strategies that Utilize Nanotechnology to Treat Cartilage Damage. Open Orthop J, 2016. 10(S3-M6): p. 862-876.

57. Roach, H.I. and S. Tilley, The Pathogenesis of Osteoarthritis in Bone and Osteoarthritis, F. Bronner and M.C. Farach-Carson, Editors. 2007, Springer-Verlag Ltd: London. p. 1-10.

58. Schmidt, T.W., Approach to Osteoarthritis Management for the Primary Care Provider. Primary Care: Clinics in Office Practice, 2018. 45(2): p. 361-378.

59. Varady, N.H., E.C. Dee, and J.N. Katz, International assessment on quality and content of internet information on osteoarthritis. Osteoarthritis and Cartilage, 2018. 26(8): p. 1017-1026.

60. Salmon, J.H., et al., Economic impact of lower-limb osteoarthritis worldwide: a systematic review of cost-of-illness studies. Osteoarthritis and Cartilage, 2016. 24(9): p. 1500-1508.

61. Nelson, A.E., Osteoarthritis year in review 2017: clinical. Osteoarthritis and Cartilage, 2018. 26(3): p. 319-325.

62. Osteoarthritis: A Serious Disease, in Osteoarthritis Research Society International. 2016, US Food and Drug Administration: USA. p. 1-103.

63. Allen, K.D. and Y.M. Golightly, Epidemiology of osteoarthritis: state of the evidence. Current opinion in rheumatology, 2015. 27(3): p. 276.

64. Johnson, V.L. and D.J. Hunter, The epidemiology of osteoarthritis. Best Practice \& Research Clinical Rheumatology, 2014. 28(1): p. 5-15.

65. Kontis, V., et al., Future life expectancy in 35 industrialised countries: projections with a Bayesian model ensemble. The Lancet, 2017. 389(10076): p. 1323-1335.

66. Dejaco, C., et al., Rheumatology Workforce Planning in Western Countries: A Systematic Literature Review. Arthritis Care \& Research, 2016. 68(12): p. 1874-1882.

67. Murphy, L.B., et al., Medical Expenditures and Earnings Losses Among US Adults With Arthritis in 2013. Arthritis Care Res (Hoboken), 2018. 70(6): p. 8.

68. Kellgren, J. and J. Lawrence, Radiological assessment of osteo-arthrosis. Annals of the Rrheumatic Diseases, 1957. 16(4): p. 494-502. 
69. Kellgren, J.H. and J.S. Lawrence, Rheumatism in miners. Part II: X-Ray study. British Journal of Industrial Medicine, 1952. 9(3): p. 197-207.

70. World Health Organization, in Burden of Musculoskeletal Conditions at the Start of the New Millennium. 2000, World Health Organization: Geneva. p. 1-231.

71. Felson, D.T., et al., The incidence and natural history of knee osteoarthritis in the elderly, the framingham osteoarthritis study. Arthritis \& Rheumatism, 1995. 38(10): p. 1500-1505.

72. Skovron, M.L., et al., Sociocultural factors and back pain. A population-based study in Belgian adults. Spine, 1994. 19(2): p. 129-137.

73. Iannone, F. and G. Lapadula, The pathophysiology of osteoarthritis. Aging Clinical and Experimental Research, 2003. 15(5): p. 364-372.

74. Bähler, C., et al., Multimorbidity, health care utilization and costs in an elderly community-dwelling population: a claims data based observational study. BMC Health Services Research, 2015. 15(1): p. 23-34.

75. Howell, D.S., Pathogenesis of osteoarthritis. The American Journal of Medicine, 1986. 80(4): p. 24-28.

76. Campisi, J. and F. d'Adda di Fagagna, Cellular senescence: when bad things happen to good cells. Nature Reviews: Molecular Cell Biology, 2007. 8(9): p. 729-740.

77. Rosenthal, A.K., Calcium crystal deposition and osteoarthritis. Rheumatic Disease Clinics, 2006. 32(2): p. 401-412.

78. Anderson, A.S. and R.F. Loeser, Why is osteoarthritis an age-related disease? Best Practice \& Research Clinical Rheumatology, 2010. 24(1): p. 15-26.

79. van Meurs, J.B.J., Osteoarthritis year in review 2016: genetics, genomics and epigenetics. Osteoarthritis and Cartilage, 2017. 25(2): p. 181-189.

80. Steinberg, J. and E. Zeggini, Functional genomics in osteoarthritis: Past, present, and future. Journal of Orthopaedic Research, 2016. 34(7): p. 1105-1110.

81. Xiao, Y.-P., et al., Are estrogen-related drugs new alternatives for the management of osteoarthritis? Arthritis Research \& Therapy, 2016. 18(1): p. 151.

82. Lohmander, L.S., et al., High prevalence of knee osteoarthritis, pain, and functional limitations in female soccer players twelve years after anterior cruciate ligament injury. Arthritis \& Rheumatism, 2004. 50(10): p. 3145-3152.

83. Felson, D.T., et al., Osteoarthritis: New insights. part 1: the disease and its risk factors. Annals of Internal Medicine, 2000. 133(8): p. 635-646.

84. Croft, P., et al., Osteoarthritis of the hip: an occupational disease in farmers. British Medical Journal, 1992. 304(6837): p. 1269-1272.

85. Hadler, N.M., et al., Hand structure and function in an industrial setting. Arthritis \& Rheumatism, 1978. 21(2): p. 210-220.

86. Jiang, L., et al., Body mass index and susceptibility to knee osteoarthritis: A systematic review and metaanalysis. Joint Bone Spine, 2012. 79(3): p. 291-297.

87. Sokoloff, L., Aging and Degenerative Diseases Affecting Cartilage, in Cartilage: Biomedical Aspects, B.K. Hall, Editor. 1983, Academic Press: New York. p. 109-141.

88. Kotecha, M., et al., Application of sodium triple-quantum coherence NMR spectroscopy for the study of growth dynamics in cartilage tissue engineering. NMR in Biomedicine, 2013. 26(6): p. 709-717.

89. Coutts, R.D., et al., Matrices for Cartilage Repair. Clinical Orthopaedics and Related Research, 2001. 391(Supplement): p. S271-S279.

90. Bert, J.M., Abandoning microfracture of the knee: Has the time come? Arthroscopy: The Journal of Arthroscopic \& Related Surgery, 2015. 31(3): p. 501-505.

91. Santo, V.E., et al., Controlled release strategies for bone, cartilage, and osteochondral engineeringPart I: Recapitulation of native tissue healing and variables for the design of delivery systems. Tissue Engineering Part B: Reviews, 2013. 19(4): p. 308-326.

92. Yousefi, A.M., et al., Current strategies in multiphasic scaffold design for osteochondral tissue engineering: A review. Journal of Biomedical Material Research. Part A, 2015. 103(7): p. 2460-81. 
93. Brittberg, M., et al., Treatment of deep cartilage defects in the knee with autologous chondrocyte transplantation. New England Journal of Medicine, 1994. 331(14): p. 889-895.

94. Becerra, J., et al., The stem cell niche should be a key issue for cell therapy in regenerative medicine. Stem Cell Reviews and Reports, 2011. 7(2): p. 248-255.

95. Brittberg, M., Cell Carriers as the Next Generation of Cell Therapy for Cartilage Repair:A Review of the Matrix-Induced Autologous Chondrocyte Implantation Procedure. The American Journal of Sports Medicine, 2010. 38(6): p. 1259-1271.

96. Dobratz, E.J., et al., Injectable cartilage: Using alginate and human chondrocytes. Archives of Facial Plastic Surgery, 2009. 11(1): p. 40-47.

97. Hollander, A.P., S.C. Dickinson, and W. Kafienah, Stem Cells and Cartilage Development: Complexities of a Simple Tissue. Stem Cells, 2010. 28(11): p. 1992-1996.

98. Labusca, L. and F. Zugun-Eloae, Stem Cell Therapy for the Treatment of Cartilage Defects and Osteoarthritis, in Stem Cells in Clinical Applications: Bone and Cartilage Regeneration, P.V. Pham, Editor. 2016, Springer International Publishing, Springer Nature: Switzerland. p. 11-46.

99. Behrens, P., et al., Matrix-associated autologous chondrocyte transplantation/implantation (MACT/MACI): 5-year follow-up. The Knee, 2006. 13(3): p. 194-202.

100. Pettersson, S., et al., Human articular chondrocytes on macroporous gelatin microcarriers form structurally stable constructs with blood-derived biological glues in vitro. Journal of Tissue Engineering and Regenerative Medicine, 2009. 3(6): p. 450-460.

101. Park, J. and R.S. Lakes, Biomaterials: an introduction. 2007, New York: Springer Science \& Business Media. 563.

102. Ratner, B.D., et al., Biomaterials science: A multidisciplinary endeavor, in Biomaterials science: An introduction to materials in medicine, B.D. Ratner, et al., Editors. 2004, Elsevier: San Diego \& London. p. 1-19.

103. Shukla, S.K., Nanostructure polymers in function generating substitute and organ transplants in Nanomaterials in Drug Delivery, Imaging, and Tissue Engineering, A. Tiwari and A. Tiwari, Editors. 2013, John Wiley \& Sons, and Scrivener Publishing LLC: Hoboken, New Jersey, and Salem, Massachusetts. p. 397-423.

104. Kim, N.J., S.J. Lee, and A. Atala, Biomedical nanomaterials in tissue engineering, in Nanomaterials in tissue engineering, A.K. Gaharwar, et al., Editors. 2013, Woodhead Publishing Limited: Sawston, Cambridge. p. 1-24.

105. Hampel, U., et al., Chemokine and cytokine levels in osteoarthritis and rheumatoid arthritis synovial fluid. Journal of Immunological Methods, 2013. 396(1): p. 134-139.

106. Scanzello, C.R., et al., Local cytokine profiles in knee osteoarthritis: elevated synovial fluid interleukin-15 differentiates early from end-stage disease. Osteoarthritis and Cartilage, 2009. 17(8): p. 1040-1048.

107. Bajpayee, A.G. and A.J. Grodzinsky, Cartilage-targeting drug delivery: can electrostatic interactions help? Nature Reviews Rheumatology, 2017. 13(3): p. 183-193.

108. da Costa, B.R., et al., Effectiveness of non-steroidal anti-inflammatory drugs for the treatment of pain in knee and hip osteoarthritis: a network meta-analysis. The Lancet, 2017. 390(10090): p. e21e33.

109. Urech, D.M., et al., Anti-inflammatory and cartilage-protecting effects of an intra-articularly injected anti-TNFa single-chain Fv antibody (ESBA105) designed for local therapeutic use. Annals of the Rheumatic Diseases, 2010. 69(2): p. 443-449.

110. Feldmann, M. and R.N. Maini, Anti-TNFa Therapy of Rheumatoid Arthritis: What Have We Learned? Annual Review of Immunology, 2001. 19(1): p. 163-196.

111. DiDomenico, C.D. and L.J. Bonassar, How can 50 years of solute transport data in articular cartilage inform the design of arthritis therapeutics? Osteoarthritis and Cartilage, 2018. XX(YY): p. ZZ-ZZ. 
112. Yang, L. and T.J. Webster, Nanotechnology controlled drug delivery for treating bone diseases. Expert Opinion on Drug Delivery, 2009. 6(8): p. 851-864.

113. Sonia, T.A. and C.P. Sharma, Chitosan and Its Derivatives for Drug Delivery Perspective, in Chitosan for biomaterials I, R. Jayakumar, M. Prabaharan, and R.A. Muzzarelli, Editors. 2011, Springer: Heidelberg. p. 23-54.

114. Cho, K., et al., Therapeutic Nanoparticles for Drug Delivery in Cancer. Clinical Cancer Research, 2008. 14(5): p. 1310-1316.

115. Guler, M.O. and A.B. Tekinay, Nanomaterials for medicine, in Therapeutic Nanomaterials, M.O. Guler and A.B. Tekinay, Editors. 2016, John Wiley \& Sons: Hoboken, New Jersey. p. 1-6.

116. Madry, H., et al., Transforming Growth Factor Beta-Releasing Scaffolds for Cartilage Tissue Engineering. Tissue Engineering Part B: Reviews, 2014. 20(2): p. 106-125.

117. Biondi, M., et al., Nanoparticle-Integrated Hydrogels as Multifunctional Composite Materials for Biomedical Applications. Gels, 2015. 1(2): p. 162.

118. Song, F., et al., Nanocomposite Hydrogels and Their Applications in Drug Delivery and Tissue Engineering. Journal of Biomedical Nanotechnology, 2015. 11(1): p. 40-52.

119. Asghari, F., et al., Biodegradable and biocompatible polymers for tissue engineering application: a review. Artificial Cells, Nanomedicine, and Biotechnology, 2017. 45(2): p. 185-192.

120. Jayaraman, P., et al., Controlled release of drugs in electrosprayed nanoparticles for bone tissue engineering. Advanced Drug Delivery Reviews, 2015. 94(11): p. 77-95.

121. Eftekhari, H., et al., Assessment of polycaprolacton (PCL) nanocomposite scaffold compared with hydroxyapatite (HA) on healing of segmental femur bone defect in rabbits. Artificial Cells, Nanomedicine, and Biotechnology, 2017. 45(5): p. 961-968.

122. Asadi, N., et al., Nanocomposite hydrogels for cartilage tissue engineering: a review. Artificial Cells, Nanomedicine, and Biotechnology, 2018. 46(3): p. 465-471.

123. Khalilov, R.I., I.S. Ahmadov, and S.G. Kadirov, Two types of kinetics of membrane potential of water plant leaves illuminated by ultraviolet light. Bioelectrochemistry, 2002. 58(2): p. 189-191.

124. Son, J.-S., et al., Development and structure of a novel barrier membrane composed of drug-loaded poly(lactic-co-glycolic acid) particles for guided bone regeneration. Biotechnology Letters, 2012. 34(4): p. 779-787.

125. Shi, J., et al., Nanoparticle Delivery of the Bone Morphogenetic Protein 4 Gene to Adipose-Derived Stem Cells Promotes Articular Cartilage Repair In Vitro and In Vivo. Arthroscopy: The Journal of Arthroscopic \& Related Surgery, 2013. 29(12): p. 2001-2011.

126. te Boekhorst, B.C.M., et al., MRI-assessed therapeutic effects of locally administered PLGA nanoparticles loaded with anti-inflammatory siRNA in a murine arthritis model. Journal of Controlled Release, 2012. 161(3): p. 772-780.

127. Whitmire, R.E., et al., Self-assembling nanoparticles for intra-articular delivery of antiinflammatory proteins. Biomaterials, 2012. 33(30): p. 7665-7675.

128. Virto, M.R., et al., Gentamicin release from modified acrylic bone cements with lactose and hydroxypropylmethylcellulose. Biomaterials, 2003. 24(1): p. 79-87.

129. Virto, M.R., et al., Improvement of gentamicin poly(d,l-lactic-co-glycolic acid) microspheres for treatment of osteomyelitis induced by orthopedic procedures. Biomaterials, 2007. 28(5): p. 877-885.

130. Torrado, S., P. Frutos, and G. Frutos, Gentamicin bone cements: characterisation and release (in vitro and in vivo assays). International Journal of Pharmaceutics, 2001. 217(1): p. 57-69.

131. Fiedler, J., et al., The effect of substrate surface nanotopography on the behavior of multipotnent mesenchymal stromal cells and osteoblasts. Biomaterials, 2013. 34(35): p. 8851-8859.

132. Shimaya, M., et al., Magnesium enhances adherence and cartilage formation of synovial mesenchymal stem cells through integrins. Osteoarthritis and Cartilage, 2010. 18(10): p. 1300-1309.

133. Guo, Z., et al., In vitro evaluation of random and aligned polycaprolactone/gelatin fibers via electrospinning for bone tissue engineering. Journal of Biomaterials Science, Polymer Edition, 2015. 26(15): p. 989-1001. 
134. Lee, K.-B., et al., Protein Nanoarrays Generated By Dip-Pen Nanolithography. Science, 2002. 295(5560): p. 1702-1705.

135. González-Henríquez, C.M., et al., Thin and ordered hydrogel films deposited through electrospinning technique; a simple and efficient support for organic bilayers. Biochimica et Biophysica Acta (BBA) - Biomembranes, 2015. 1848(10, Part A): p. 2126-2137.

136. Maudens, P., O. Jordan, and E. Allémann, Recent advances in intra-articular drug delivery systems for osteoarthritis therapy. Drug Discovery Today, 2018. 23(10): p. 1761-1775.

137. He, Z., et al., An overview of hydrogel-based intra-articular drug delivery for the treatment of osteoarthritis. Colloids and Surfaces B: Biointerfaces, 2017. 154: p. 33-39.

138. Gerwin, N., C. Hops, and A. Lucke, Intraarticular drug delivery in osteoarthritis. Advanced Drug Delivery Reviews, 2006. 58(2): p. 226-242.

139. Kang, M.L. and G.-I. Im, Drug delivery systems for intra-articular treatment of osteoarthritis. Expert Opinion on Drug Delivery, 2014. 11(2): p. 269-282.

140. Rivera-Delgado, E., et al., Injectable liquid polymers extend the delivery of corticosteroids for the treatment of osteoarthritis. Journal of Controlled Release, 2018. 284: p. 112-121.

141. Ryan, S.M., et al., An intra-articular salmon calcitonin-based nanocomplex reduces experimental inflammatory arthritis. Journal of Controlled Release, 2013. 167(2): p. 120-129.

142. Morgen, M., et al., Nanoparticles for Improved Local Retention after Intra-Articular Injection into the Knee Joint. Pharmaceutical Research, 2013. 30(1): p. 257-268.

143. Horisawa, E., et al., Prolonged Anti-Inflammatory Action of DL-Lactide/Glycolide Copolymer Nanospheres Containing Betamethasone Sodium Phosphate for an Intra-Articular Delivery System in Antigen-Induced Arthritic Rabbit. Pharmaceutical Research, 2002. 19(4): p. 403-410.

144. Saadat, E., et al., Hyaluronic acid based micelle for articular delivery of triamcinolone, preparation, in vitro and in vivo evaluation. International Journal of Pharmaceutics, 2015. 489(1): p. 218-225.

145. Elron-Gross, I., Y. Glucksam, and R. Margalit, Liposomal dexamethasone-diclofenac combinations for local osteoarthritis treatment. International Journal of Pharmaceutics, 2009. 376(1): p. 84-91.

146. Maudens, P., et al., Nanocrystal-Polymer Particles: Extended Delivery Carriers for Osteoarthritis Treatment. Small, 2018. 14(8): p. 1703108.

147. Kang, M.L., et al., Intra-articular delivery of kartogenin-conjugated chitosan nano/microparticles for cartilage regeneration. Biomaterials, 2014. 35(37): p. 9984-9994.

148. Kang, M.-L., J.-E. Kim, and G.-I. Im, Thermoresponsive nanospheres with independent dual drug release profiles for the treatment of osteoarthritis. Acta Biomaterialia, 2016. 39: p. 65-78.

149. Jain, A., et al., Targeting of diacerein loaded lipid nanoparticles to intra-articular cartilage using chondroitin sulfate as homing carrier for treatment of osteoarthritis in rats. Nanomedicine: Nanotechnology, Biology and Medicine, 2014. 10(5): p. e1031-e1040.

150. Bartlett, R.L., S. Sharma, and A. Panitch, Cell-penetrating peptides released from thermosensitive nanoparticles suppress pro-inflammatory cytokine response by specifically targeting inflamed cartilage explants. Nanomedicine: Nanotechnology, Biology and Medicine, 2013. 9(3): p. 419-427.

151. Lin, J.B., S. Poh, and A. Panitch, Controlled release of anti-inflammatory peptides from reducible thermosensitive nanoparticles suppresses cartilage inflammation. Nanomedicine: Nanotechnology, Biology and Medicine, 2016. 12(7): p. 2095-2100.

152. Lu, H., et al., Chitosan-Graft-Polyethylenimine/DNA Nanoparticles as Novel Non-Viral Gene Delivery Vectors Targeting Osteoarthritis. PLOS ONE, 2014. 9(1): p. e84703.

153. Huang, X., et al., Cross-linked polyethylenimine-tripolyphosphate nanoparticles for gene delivery. International journal of nanomedicine, 2014. 9: p. 4785-4794.

154. $\mathrm{Pi}, \mathrm{Y}$., et al., Intra-articular delivery of anti-Hif-2 $\alpha$ siRNA by chondrocyte-homing nanoparticles to prevent cartilage degeneration in arthritic mice. Gene Therapy, 2015. 22: p. 439.

155. Park, J.S., et al., The use of anti-COX2 siRNA coated onto PLGA nanoparticles loading dexamethasone in the treatment of rheumatoid arthritis. Biomaterials, 2012. 33(33): p. 8600-8612.

156. Evans, C.H., et al., Osteoarthritis gene therapy. Gene Therapy, 2004. 11: p. 379. 
157. Drury, J.L. and D.J. Mooney, Hydrogels for tissue engineering: scaffold design variables and applications. Biomaterials, 2003. 24(24): p. 4337-4351.

158. Lu, S. and K.S. Anseth, Release Behavior of High Molecular Weight Solutes from Poly(ethylene glycol)-Based Degradable Networks. Macromolecules, 2000. 33(7): p. 2509-2515.

159. Lim, H.J., et al., Controlled release of BMP-2 from alginate nanohydrogels enhanced osteogenic differentiation of human bone marrow stromal cells. Macromolecular Research, 2010. 18(8): p. 787792.

160. Sehgal, R.R. and R. Banerjee, Fabrication of nanomaterials for growth factor delivery in tissue engineering, in Nanomaterials in tissue engineering, A.K. Gaharwar, et al., Editors. 2013, Woodhead Publishing Limited: Sawston, Cambridge. p. 183-226.

161. Pan, Y.-J., et al., Redox/pH dual stimuli-responsive biodegradable nanohydrogels with varying responses to dithiothreitol and glutathione for controlled drug release. Biomaterials, 2012. 33(27): p. 6570-6579.

162. Zhang, J., et al., The targeted behavior of thermally responsive nanohydrogel evaluated by NIR system in mouse model. Journal of Controlled Release, 2008. 131(1): p. 34-40.

163. Li, J., et al., Self-assembly of DNA Nanohydrogels with Controllable Size and Stimuli-Responsive Property for Targeted Gene Regulation Therapy. Journal of the American Chemical Society, 2015. 137(4): p. 1412-1415.

164. Iwasa, J., et al., Clinical application of scaffolds for cartilage tissue engineering. Knee Surgery, Sports Traumatology, Arthroscopy, 2009. 17(6): p. 561-577.

165. Kerker, J.T., A.J. Leo, and N.A. Sgaglione, Cartilage Repair: Synthetics and Scaffolds: Basic Science, Surgical Techniques, and Clinical Outcomes. Sports Medicine and Arthroscopy Review, 2008. 16(4): p. 208-216.

166. Temenoff, J.S. and A.G. Mikos, Review: tissue engineering for regeneration of articular cartilage. Biomaterials, 2000. 21(5): p. 431-440.

167. Ferruzzi, A., et al., Autologous Chondrocyte Implantation in the Knee Joint: Open Compared with Arthroscopic Technique: Comparison at a Minimum Follow-up of Five Years. JBJS, 2008. 90(Supplement_4): p. 90-101.

168. Lien, S.-M., L.-Y. Ko, and T.-J. Huang, Effect of pore size on ECM secretion and cell growth in gelatin scaffold for articular cartilage tissue engineering. Acta Biomaterialia, 2009. 5(2): p. 670-679.

169. Gerard, C., et al., The effect of alginate, hyaluronate and hyaluronate derivatives biomaterials on synthesis of non-articular chondrocyte extracellular matrix. Journal of Materials Science: Materials in Medicine, 2005. 16(6): p. 541-551.

170. Raghunath, J., et al., Biomaterials and scaffold design: key to tissue-engineering cartilage. Biotechnology and Applied Biochemistry, 2007. 46(2): p. 73-84.

171. Awad, H.A., et al., Chondrogenic differentiation of adipose-derived adult stem cells in agarose, alginate, and gelatin scaffolds. Biomaterials, 2004. 25(16): p. 3211-3222.

172. Steinert, A., et al., Chondrogenic differentiation of mesenchymal progenitor cells encapsulated in ultrahigh-viscosity alginate. Journal of Orthopaedic Research, 2003. 21(6): p. 1090-1097.

173. $\mathrm{Ng}, \mathrm{K} . \mathrm{W}$., et al., A layered agarose approach to fabricate depth-dependent inhomogeneity in chondrocyte-seeded constructs. Journal of Orthopaedic Research, 2005. 23(1): p. 134-141.

174. Shelton, J.C., D.L. Bader, and D.A. Lee, Mechanical Conditioning Influences the Metabolic Response of Cell-Seeded Constructs. Cells Tissues Organs, 2003. 175(3): p. 140-150.

175. Pieper, J.S., et al., Crosslinked type II collagen matrices: preparation, characterization, and potential for cartilage engineering. Biomaterials, 2002. 23(15): p. 3183-3192.

176. Roche, S., et al., Native and DPPA cross-linked collagen sponges seeded with fetal bovine epiphyseal chondrocytes used for cartilage tissue engineering. Biomaterials, 2000. 22(1): p. 9-18.

177. Ponticiello, M.S., et al., Gelatin-based resorbable sponge as a carrier matrix for human mesenchymal stem cells in cartilage regeneration therapy. Journal of Biomedical Materials Research, 2000. 52(2): p. 246-255. 
178. Quintavalla, J., et al., Fluorescently labeled mesenchymal stem cells (MSCs) maintain multilineage potential and can be detected following implantation into articular cartilage defects. Biomaterials, 2002. 23(1): p. 109-119.

179. Dragoo, J.L., et al., Tissue-engineered cartilage and bone using stem cells from human infrapatellar fat pads. The Journal of Bone and Joint Surgery. British volume, 2003. 85-B(5): p. 740-747.

180. Peretti, G.M., et al., Cell-Based Tissue-Engineered Allogeneic Implant for Cartilage Repair. Tissue Engineering, 2000. 6(5): p. 567-576.

181. Nawaz, M., Nanotechnology-Based Approaches in Pediatric Parasitic Infections. Journal of Pediatric Infectious Diseases, 2017. 12(04): p. 264-270.

182. Shyam, S. and P. Vijay Kumar, Drug Targeting to Infectious Diseases by Nanoparticles Surface Functionalized with Special Biomolecules. Current Medicinal Chemistry, 2012. 19(19): p. 3196-3202.

183. Elsabahy, M. and K.L. Wooley, Cytokines as biomarkers of nanoparticle immunotoxicity. Chemical Society Reviews, 2013. 42(12): p. 5552-5576. 